Ueber die Geometrie der Kegelschnitte, insbesondere deren Charakteristikenproblem.

Von

E. Study in Leipzig.

Bei der grundlegenden Bedeutung, welche das Bé zout'sche Theorem für die meisten geometrischen Untersuchungen besitzt, war es natürlich, dass die Geometer, sobald sie überhaupt anfingen, neben den früher allein untersuchten Curven und Oberflächen auch zusammengesetztere Gebilde in den Kreis ihrer Betrachtung zu ziehen, danach strebten, jenes mächtige Werkzeug auf das neu erschlossene Arbeitsfeld mit sich zu nehmen, also den Bézout'schen Satz auf jene Gebilde auszudehnen, beziehungsweise sein Analogon aufzusuchen.

Eine der ersten und wichtigsten Verallgemeinerungen, welche so die Bézout'sche Fragestellung erfuhr, bezog sich auf Systeme von Curven und Oberflächen.

Es war, allgemein zu reden, das Problem: „Diejenigen Curven oder Flächen eines (algebraischen) Systems solcher Gebilde anzugeben, welche gegebenen Bedingungen genügen"; oder, mit anderen Worten: "Hehrere in ihren Dimensionen einander auf geeignete Weise ergänzende Systeme von Curven oder Flächen gleicher Art zum Durchschnitt zu bringen", d. b. die Gleichung anzugeben, von deren lösung die Auffindung derselben in letzter Instanz abhängt, oder doch wenigstens den Grad der Gleichung, die Anzahl der Lösungen oder die Ordnung des Lösungssystems zu bestimmen.

Der erste, welcher die letztere Aufgabe - in der es sich also lediglich um eine Erweiterung der Bezout'schen Sätze handelte - in ihrer Allgemeinheit augriff, oder doch anzugreifen glaubte, war de J onquières.

Seine Beantwortung der Frage bezog sich allerdings nur auf einen besonderen Fall - den er noch für den allgemeinen ansah -, man 
kann ihr aber, mit Bezugnahme auf eine der Folgezeit geläufig gewordene Anschaunngsweise, einen generellen Ausdruck geben, dahin lautend, dass diese erweiterte Aufgabe noch in das Gebiet des Bézout'schen Theorems selbst fällt.

$\mathrm{Da}$ nämlich beispielsweise alle ebenen Curven $n^{\text {ter }}$ Ordnung eine lineare Marnichfaltigkeit bilden, und man diese auf eine lineare Punktmannichfaltigkeit ein-eindeutig beziehen kann, so ist die Frage ohne Weiteres auf Bézout's originale Problemstellung zurückführbar.

Mit Rücksicht auf die vorhergenannte Aufgabe wird also, zufolge der ursprünglichen Auffassung de Jonquières', jedes System von Curven derselben Ordnung in der Ebene durch eine einzige Zahl charakterisirt, den "Index" des Systems, welcher der Ordnung etwa einer Raumcurve oder einer Fläche analog ist; und man erhält die Anzahl der mehreren Systemen von geeigneten Dimensionen gemeinsamen Curven, indem man das Product ihrer Indices bildet.

Dieser Standpunkt zeigt nun offenbar den Bézout'schen Sätzen gegenüber nichts eigentlich Neues; die Theorie der Systeme algebraischer Curven selbst aber lässt er in einem falschen Lichte erscheinen, wie man schon daraus erkennen kann, dass man vou hier zu Unterscheidungen gelangt, die in der Natur jener Gebilde nicht begründet sind.

Es beruht dies auf der Auszeichnung des Punktes als Raumelement. Eine Curve ist aber nicht allein Ort von Punkten; und man wird etwa ein Kegelschnittsystem nicht nur als ein System von Curven zweiter Ordnung, also von Punkteurven betrachten dürfen, sondern man wird dasselbe ebensowohl als ein System von Curven zweiter Classe aufzufassen haben, d. h. als ein System von Oertern für gerade Linien.

Fin und dasselbe Gebilde erhält aber in beiden Fällen verschiedene analytische Darstellungen und zugleich verschiedene Indices; man muss daher, wenn man sich nicht in Widersprüche verwickeln will, beide Standpunkte streng auseinanderhalten*), wozu im Begriffe des Kegelschnittes kein Grund liegen kann.

Es bedeutete mithin einen grossen Fortschritt in dieser Theorie, als Chasles gegen de Jonquières geltend machte, dass von der Gesammtzahl jener Lösungen gewisse in Abzug zu bringen seien, welche

*) Während man nämlich zuerst zwei Curven als identisch ansah, wenn sie Punkt für Punkt übereinstimmten, bat man sie jetzt als identisch zu betrachten, wenn ihre Tangenten dieselben sind. Das eine ist aber nicht immer eine Folge des anderen, nämlich bei ausgearteten Curven nicht; und dadurch eben nnterscheiden sich jene beiden Anffassungsweisen, dass man in jedem Falle andere ausgeartete Curven noch mit zu dem System rechnet. In beiden Fällen begeht man offenbar einen Act der Willkïr; ihn zu vermeiden, ohne - wie es geschehen ist - in einen ähnlichen Fehler zu verfallen, ist das punctum saliens bei diesem Problem. 
den Bedingungen der Aufgabe zwar selbstverständlich, aber doch nicht. eigentlich genügten *).

Suchte man nämlich beispielsweise die Zahl der Curven 2. 0 . eines einfach ausgedehnten Systems, welche eine beliebig gegebene Curve berühren, so erhielt man, wenn sich doppelt zählende Gerade nnter den Kegelschnitten des Systems befanden, dieselben immer mit als Lösungen der Aufgabe.

Diese ausgearteten Kegelschnitte erfüllten aber die Bedingungen des Problems offenbar in ganz anderer Weise als die übrigen, im Allgemeinen nicht ausgearteten Kegelschnitte der Reihe, welche jene Curve berühren im gewöhnlichen Sinne des Wortes, und waren daher, da man sich nur für die letzteren interessirte, nicht mitzuzählen.

So gewendet, war die Frage nun nicht mehr so lejcht allgemein zu behandeln, wie in der früheren Fassung; doch hatte Chasles die Antwort wenigstens für Systeme von Kegelschnitten einer und derselben Ebene durch Induction gefunden: Er stellte den Satz auf, dass die Zahl der Kegelschnitte, in welchen sich ein einfach- und ein vierfachausgedehntes System durchdringen, durch einen Ausdruck von der Form

$$
\alpha \mu+\beta \nu
$$

angegeben werde, worin $\alpha, \beta, \mu, \nu$ Zahlen sind, von welchen $\mu$ und $\nu$ für das èrste System "charahteristisch" und von dem zweiten ganz unabhängig sind, während für $\alpha$ und $\beta$ das Omgekehrte gilt.

Aus dieser Formel leitete Chasles noch eine zweite ab, welche die Zahl der Kegelschnitte, in welchen sich fünf beliebige, vierfach ausgedehnte Systeme treffen, als eine lineare Funktion der beiden "Charakteristiken" $\alpha, \beta$ eines jeden derselben angab, und Cremona fügte der Chasles'schen eine ähnlich gebaute Formel hinzu, welche sich auf die Gegenüberstellung eines zweifach und eines dreifach ausgedehnten Systems bezog, die nun nicht mehr Durchschnitte von drei, bezüglich zwei vierfach ausgedehnten Systemen zu sein brauchten. (Alles dieses in den Comptes Rendus des Jahres 1864.)

Obwohl nun diese Sätze um ihrer merkwürdigen Form, ihrer Allgemeinheit und grossen Fruchtbarkeit willen das lebhafteste Interesse der Geometer erregten, und man dieselben nicht allein in zahlreichen Beispielen bestätigt fand (besonders bei Berührungsaufgaben), sondern auch - freilich nur mit sehr beschränktem Erfolg den Versuch machte, dieselben auf höhere Gebilde auszudehnen, so erschien doch erst nach Verlauf von fast einem Decennium eine Abhandlung, welche die Allgemeingiltigkeit wenigstens des Chasles'schen

*) Ich drücke mich mit Absicht so unbestimmt aus, da - was $\mathrm{zq}$ bemerken für das Folgende wesentlich ist-scharfe Definitionen damals in der That nicht gegeben warden. 
Satzes zu beweisen unternahm, aus der Feder von Clebsch (Math. Annalen Bd. 6 (1873) S. 1), worauf dann bald mehrere andere Beweisversuche folgten.

Das Resultat dieser Untersuchung, die mit Hilfe der Methoden der Invariantentheorie geführt wurde, ist bekanntlich nicht richtig.

Da diese Einleitung natürlich nicht darauf ausgehen kann, die Entwickelung der umfangreichen, fast von Anfang an schon mit einem eigenen Namen bezeichneten "Theorie der Charakteristiken" genau zu verfolgen, sondern nur soviel aus derselben hervorheben will, als dem Verfasser zur Motivirung seiner eigenen Bestrebungen nothweñdig erscheint, so ist hier nicht der Ort, die Leistungen Clebsch's und seiner Nachfolger zu zergliedern. Doch müssen wir einen Umstand näher in's Auge fassen, der von nun an immer dentlicher hervortritt, und für die Auslegung des Chasles'schen Satzes in der Folge massgebend geworden ist. Es vollzog sich nämlich, fast unmerklich, eine Verschiebung der ursprünglichen Problemstellung - so unmerklich, dass dieselbe sogar von ihren Urhebern nicht beachtet worden zu sein scheint.

Den entscheidenden Schritt in dieser Richtung that Clebsch in der eben erwähnten Abhandlung, indem er derselben einen Begriff der „eigentlichen Lösung" zu Grunde legte, der bis dahin noch nicht hervorgetreten war. Die Veranlassung hierzu gab die im Vergleich zu den früheren Arbeiten abstracte Natur seiner Untersuchung.

Wir haben schon angedeutet, dass die älteren Autoren sich bei ihrer Abscheidung gewisser Arten von Lösungen mehr durch einen richtigen Tact, als durch scharfe Begriffe hatten leiten lassen. Bei den von ihnen behandelten besonderen Classen von Aufgaben lehrte der Augenschein (s. obiges Beispiel), welche von den durch das Bézont'sche Theorem, resp. durch das sogenannte "Correspondenzprincip" gelieferten Lösungen $\mathrm{zu}$ entfernen, bezüglich als "eigentliche“ beizubehalten waren; man wandte daher einer genauen, alle denkbaren Fälle umfassenden Definition dieser letzteren wenig Aufmerksamkeit zu, oder gab vielmehr gar keine Definition, da man kein Bedürfniss nach einer solchen empfand.

Will man aber eine in besonderen Fällen anschauungsmässig behandelte Aufgabe in voller Allgemeinheit erledigen, so muss man von der Anschaunng zu Begriffen übergehen und logische Deductionen an Stelle der Berufung auf den Augenschein setzen, die nicht selten auch im einzelnen Falle, wo sie auszureichen scheint, doch nur das stille Bekenntniss enthält, dass man sich der wahren Gründe nicht vollkommen klar bewusst ist. Man hat zu diesem Behufe diejenigen Eigenschaften der betreffenden Gebilde, deren Vorbandensein man als die nothwendige und ausreichende Bedingung ansieht für das Bestehen jener 
anderen Eigenschaften, welche den geometrischen Satz vorstellen, von den übrigen zu trennen, welche man als Consequenzen jener oder nur als durch die zufällige Erscheinungsform des allgemeinen Satzes bedingt betrachtet. Die ersteren muss man zum Range von Definitionen erbeben und zur Grundlage der Beweisführung machen.

Diese Operation vollzieht Jeder, der eine Verallgeneinerung vornimmt, mit Absicht oder auch unwillkürlich. Da sie in der Hauptsache nur in der klareren Auffassung des für einen Satz Wesentlichen besteht, so ist zur Ausführung derselben nicht nothwendig, dass sie als ein Fortschritt des Gedankens in das Bewusstsein fällt. Es kann eintreten, dass der Schnitt, welcher Wesentliches von Zufälligem trennen soll, an der falschen Stelle geführt wird, und dass die angestrebte Schärfe der Definition doch nicht ganz erreicht wird.

Dies alles traf nun im vorliegenden Falle zu, wo es sich darum handeln musste, das "offenbar" zu analysiren, welchem zufolge nur gewisse Lösungen ais eigentliche angesehen werden durften.

Es wird zwar bei Clebsch, wie bei seinen Nachfolgern, der Chaslessche Satz immer noch in der alten Weise ausgesprochen: Es wird schlechthin von Kegelschnitten geredet, welche eine gegebene Bedingung erfüllen, und eine Definition für das Erfülltsein einer Bedingung wird dem Satze nicht vorangestellt, wiewohl eine solche hinsichtlich der ausgearteten Kegelschnitte nicht überflüssig ist.

Betrachten wir aber den Beweisgang jener Abhandlung, so sehen wir, dass ihr Verfasser ausschliesslich die bevoeglichen Curven als die eigentlichen Lösungen des Problems betrachtete, so dass wir also hier eine bestimmtere Auffassung des Satzes vor uns haben, als man dem Wortlaute nach viermuthen sollte.

Es bedarf dies noch einer kurzen Erläuterung.

Während in den bis dahin behandelten Classen von Aufgaben bei Zusammenstellung eines einfach und eines vierfach ausgedehnten Systems die "uneigentlichen" Lösungen ihren Ort in dem ersteren System unverändert beibehielten, wenn man die relative Lage beider Systeme durch collineare Transformation des einen änderte (vgl. wieder obiges Beispiel), hatten die anderen volle Beweglichkeit, was sich analytisch dadurch kund gab, dass die ersteren die das vierfach ausgedehnte $\mathrm{Sy}$ stem darstellende Bedingungsgleichung identisch befriedigten, die letzteren nicht.

Hierin erblickte Clebsch offenbar einen wesentlichen Unterschied beider Arten von Lösungen*) und dem entsprechend war seine Auf-

*) Den scheinbar näher liegenden Umstand, dass diie uneigentlichen Lösungen .ausgeartete Curven sind, für diese Unterseheidung zu benatzen, ging nicht an, da nnter Umständen auch Lösungen ansarten können, die ganz sicher den Cha- 
fassung des Chasles'schen Satzes. Damit aber, dass der Begriff der eigentlichen Lösung (ein Wort, das Clebsch übrigens nicht gebraucht) ausschliesslich an die Beweglichtieit derselben geknüpft wurde, war dieser Satz selbst, wenn auch nicht dem Wortlaut, so doch dem Sinne nach schärfer als zwor gefasst, gleichzeitig aber war etwas in die Formulirung der zu demselben führenden Problemstellung hineingetragen, das ursprïnglich nicht darin gèlegen hatte.

Dass man aber hiermit, nicht mehr genau den Chasles'schen Satz selbst vor sich hatte, sondern nur eine einseitige Ansicht desselben, neben der vielleicht auch noch andere denkbar sind, wurde übersehen. Dieser scheinbar geringfügige Umstand hat später dazu geführt, 'dass der Chasles'sche Satz als vollständig falsch bezeichnet worden ist.

Die nach Clebsch's Abhandlung erschienenen Untersuchungen können wir hier nur kurz berübren, da es uns nur auf die Auffassung der Problemstellung und des Chasles'schen Theorems ankommt, und die Folgezeit in dieser Richtung einen wesentlichen Fortschritt nicht gemacht hat. Es verharren diese Arbeiten nämlich entweder auf dem früberen Standpunkt - lassen also auch implicite keinen schärferen Begriff der eigentlichen Lösungen erkennen (was nicht auffallend erscheinen kann, da ein solcher auch bei Clebsch nur verdeckt eingeführt worden war), oder sie betrachten, wie die von Clebsch, nur die beweglichen Lösungen als die eigentlichen. In beiden Fällen war das Ergebniss die Formel von Chasles.

Herr Halp hen hat das Verdienst, die in der letzterwähnten Richtung gewonnenen Resultate - die einzigen ihm in exacter Formulirung vorliegenden - als unrichtig nachgewiesen und verbessert zu baben. Herr Halphen richtet zwar seine Angriffe gegen den Chasles'schen Satz im Allgemeinen, ohne einschränkenden Zusatz (zuerst in den Comptes Rendues vom 4. Sept. 1876), er hat aber offenbar nur die an die Beweglichkeit der Lösungen geknüpfte Fassung desselben im Auge. In der That zeigt sein Beispiel nur, dass die Zahl der beveg. lichen, zwei Systemen erster und vierter Dimension gemeinsamen Curven unter Umständen kleiner sein kann, als die Chasles'sche Zahl.

rakter von eigentlichen haben. - In seinem Beweise benutzt Clebsch ansser collinearen Aenderungen auch solche, welche wesentliche Constante des vierfach ansgedehnten Systems betreffen. Es kann nicht geleugnet werden, dass durch die auf nicht gerechtfertigten Voraussetzangen beruhende Hereinziehung solcher Aenderungen einige Unklarheit in den Begriff der Beweglichkeit bei Clebsch kommt. Wir dürfen aber, da die entgegengesetzte Annahme geradezn absurd ist, doch nicht zweifeln, dass Clebsch im Grunde nar die collinearen Aendernngen im Ange gehabt habe (wie denn auch die späteren Autoren nur solche in Betracht zogen), and haben also in der Herbeiziehung wesentlicher Constantenänderungen nur einen, in diesem Falle allerdings nicht unschädlichen Ueberfluss zu erblicken. 
In späteren Aufsätzen gab Herr Halphen für die richtige Zahl der beweglichen Lösungen zwar keinen geschlossenen Ausdruck an, wie der Chasles'sche war (was überbaupt nicht möglich ist), aber doch ein einfaches Verfahren, dieselbe zu bestimmen. Die Cbasles'sche Formel erwies sich also in diesem Sinne nicht als allgemeingiltig; doch gibt es, wie Herr Halphen gleichfalls zeigte, eine grosse Classe von Fällen, in denen dieselbe keiner Reduction 'bedürftig ist. (Journal de l'Ecole normale polytechnique. 1878 . t. 45.)

Dies ist im Wesentlichen noch der gegenwärtige Standpunkt der Sache.

Es hatte also - wenn ich nun zusammenfassen darf - Chasles erkannt, dass nicht alle von de Jonquières angegebenen Curven als eigentliche Lösungen der Aufgabe zu betrachten seien, zwei Systeme von Curven zum Durchschnitt zu bringen. Er hatte ferner einen Ausdruck für die Zahl dieser eigentlichen Lösungen in dem Falle aufgestellt, woo ein einfach- und ein vierfach ausgedehntes Kegelschnittsystem einander gegenüberstehen, nicht aber zugleich eine hinreichende Definition derselben gegeben. Seit Clebsch wurde dann der von Chastes ausgesprochene Satz so aufgefasst, dass man als die in demselben beibehaltenen, eigentlichen Lösungen die beweglichen betrachtete. Herr Halphen hat gezeigt, dass der Ausdruck für die Zahl dieser letzteren Lösungen nicht allgemein die Chasles'sche Formel ist.

Aus dem bisher Gesagten geht einerseits hervor, dass die Deutung des Chastes'schen Satzes, welche das Hauptgewicht auf die Beweglichkeit der Lösungen legte, keine glückliche war; andxerseits aber auch, dass die gegen diesen Satz gerichteten Angriffe im Grunde nicht ihn selbst, sondern eben nur jene besondere Auffassung treffen.

Man kann daher die Frage aufwerfen, ob man den Satz von Chasles nicht auf eine andere Art interpretiren kann, welche demselben eine ausnahmslos giltige Deutung verleiht.

Nachzuweisen, dass dem in der That so ist, ist der Hauptzweck der vorliegenden Arbeit.

Die Veranlassung zur Entstehung derselben gab zum Theil die Bemerkung, dass die an die Beweglichkeit geknüpfte Definition der beizubehaltenden Lösungen eine willkürliche ist. Betrachtet man ja auch sonst in der Geometrie z. B. einen Schnittpunkt einer Curve und einer Oberfläche darum nicht weniger als einen Schnittpunkt, weil er etwa durch gewisse Bedingungen an einen gegebenen Punkt, oder eine feste Curve gebunden ist. Dagegen ist es sonst allgemein üblich, eine 
Gebilde, von dem es zweifelhaft sein kann, ob es zu einer gegebenen Mannichfaltigkeit gehört oder nicht, dann und nur dann zu derselben zu recknen, wenn es sich (durch analytische Fortsetzung) aus unzweifelhaft dieser Mannichfaltigkeit angehörigen Gebilden stetig ableiten lässt.*)

Nähert man sich nun einem in eine Doppellinie ausgearteten Kegelschnitte in einer einfach ansgedehnten Kegelschnittreihe, so geht der Kegelschnitt, im Begriffe auszuarten, nicht schlechthin in jene Doppellinie über, sondern er plattet sich zuvor $a b$, und es entstehen auf der doppelt überdeckten Geraden zwei getrennte oder zusammenfallende Verzweigungspunkte. Erst mit diesen zusammen kann die ausgeartete Curve als ein vollständiger Kegelschnitt angesehen werden. Auf jeder doppelt überdeckten Geraden liegen aber $\infty^{2}$ Punktepaare, deren jedes mit ihr zusammen einen ausgearteten Kegelschnitt vorstellt; und von allen diesen Kegelschnitten werden wir, dem eben ausgesprochenen Princip gemäss, nur einen einzigen zu dem gegebenen System rechnen dürfen.

Dies ist auch die allgemein verbreitete Anschauung. Dass man aber dieselbe Betrachtung auch auf mehrfach ausgedehnte Kegelschnittsysteme anwenden müsse, scheint nicht anerkannt zu sein. Die Ursache dieser Inconsequenz glaube ich in Folgendem zu finden.

Es liege etwa ein vierfach ausgedehntes Kegelschnittsystem vor, welches eine Doppellinie enthält. Dann wird man sich dieser Doppellinie in dreifach unendlich vielen Fortschreitungsrichtungen (Kegelschnittbüscheln) nähern können, die in erster Annäherung dem gegebenen System angehören. Jede derselben führt durch Grenzübergang zu einem Punktepaar der Doppellinie, und man erhält auf diese Weise, da es nur $\infty^{2}$ solcher Punktepaare gibt, im Allgemeinen so scheint es wenigstens - jedes Punktepaar noch unendlich oft.

Es erscheint also beinahe selbstrerständlich, dass ein vierfach ausgedehntes Kegelschnittsystem zugleich mit einer Doppellinie alle Kegelschnitte enthält, die in Punktepaare dieser Geraden ausgeartet sind,

*) So allein nämlich erhält man allgemein giltige Sätze.

Man lässt z. B. eine Gerade, welche durch einen Doppelpunkt einer ebenen Curve geht, im Allgemeinen nicht als eine Tangente derselben gelten, weshalb man von der Erniedrignng der Classe einer Curve durch das Auftreten von Doppelpankten spricht. Hat man dagegen in einer einfach ausgedehnten Reihe von Curyen, nnter welchen sich einzelne Curven mit Doppelpunkt befinden, diejenigen Curven za bestimmen, welche eine gegebene Gerade berühren, und geht diese Gerade durch einen solchen isolirten Doppelpunkt, so wird man diesen Umstand als zufällig ansehen, und die zugehörige Curve sehr wohl als eine Berührungscurve der Geraden betrachten - nicht aber hinwiederum solche Curven, welche zu Doppelpunkten gehören, die in einer ganzen Reihe ron Curven des Systems angehörenden Doppelpunkten liegen - Alles aus dem im Texte angegebenen Grunde. 
es scheint die ganze Grenzbestimmung überflüssig zu sein, und was das Charakteristikenproblem anlangt, so scheint keine andere Formulirung desselben übrig zu bleiben, als die an die Beweglichkeit der Lösungen geknüpfte.

Es ist aber schon an einfachen. Beispielen (worunter das schon mehrfach herbeigezogene System aller Kegelschnitte, welche eine gegebene Curve berühren) zu sehen, dass dies nicht richtig ist.

Man erhält vielmebr gerade im allgemeinen Falle, trotzdem man sich jener Doppellinie aus $\infty^{3}$ Richtungen nähern kann, doch nur $\infty^{1}$ verschiedene Grenzlagen.

Stellt man non ein einfach- und ein vierfach ausgedehntes Kegelschnittsystem zusammen, von welchen das erstere eine Doppelliuie enthält, so wird diese im Allgemeinen auch in dem letzteren System entbalten sein; es entsteht durch die bezüglichen Grenzübergänge im ersten Falle ein einziges Punktepaar auf der Geraden, im zweiten eine Reihe von $\infty^{1}$ solchen, unter welchen das erste im Allgemeinen sich nicht befindet. Dieses Punktepaar wird man also nicht als eine beiden Systemen gemeinsame Curve ansehen dürfen.

Bringt man nun die nach Abzug aller derartigen Vorkommnisse noch übrig bleibenden, nun beiden Systemen wirkilich gemeinsamen Curven mit der richtigen Multiplicität in Anschlag, so wird ihre Gesammtzahl durch die Chasles'sche Formel angegeben - man erhält also eine ebenso einfache, als ungesuchte Deutung des Chasles'schen Satzes.*)

Die Bestimmnng der Multiplicität einer Lösung im einzelnen Falle bedarf natürlich einer eingehenden Untersuchung.

Was nun die Durchführung der Untersuchung anlangt, so wäre es nicht schwierig gewesen, die hier entwickelten geometrischen Sätze ohne allen analytischen Apparat abzuleiten, unter blosser Zuhilfenahme des Bézout'schen Theorems und ausgehend von der geometrisch evidenten Thatsache, dass ein Kegelschnitt vollkommen bestimmt ist, sobald er entweder als irreducible Curve oder als Punktepaar oder als Linienpaar gegeben vorliegt.

Ich habe es aber vorgezogen, die Aufgabe als einen Gegenstand der Formentheorie zu behandeln, da, nuchdem die früheren Versuche als gescheitert angesehen werden mïssen, diese allein - nicht nur wegèn des gegenwärtigen Zustandes der Wissenschaft, sondern auch aus inneren Gründen - die Möglichkeit der Verallgemeinerung des Problems auf

*) Es zeigt sich, dass diese Lösungen unter Umständen nur zum Theil bewaglich sind. Die Angabe der Zahl dieser Curven, der "eigentlichen Lösungen" Clebsch's ist dann das, was Clebsch angestrebt und Herr Halphen angeführt hat. - 
Formen von beliebig hoher Ordnung zeigt, es also wïnschenswerth erscheinen muss, auch die quadratischen Formen bereits in dieses erweiterte Gesichtsfeld zu rücken.

Betrachtet man zwei algebraische Formen oder Systeme solcher als identisch, wenn sie in ihren sämmtlichen ${ }^{*}$ ) covarianten Bildungen übereinstimmen, wobei man sich diejenigen, welche für die vorliegenden besonderen Formen identisch verschwinden, als durch Grenzübergänge bestimmt denkt - wie dies im $\S 1$ in etwas allgemeinerer Weise näher ausgeführt ist - so wird man eine zwei oder mehreren Systemen zugleich angehörige Form, bei weleher sich die Uebereinstimmung nicht auf alle covarianten Bildungen erstreckt, als jenen Systemen nicht wirklich, sondern böchstens scheinbar zugleich angehörig anzusehen haben - scheinbar nämlich, insofern man nur einen Theil der covarianten Bildungen im Auge hat.

Die Bestimmung der Zahl der beiden Systemen wirklich gemeinsamen Formen, bei welchen also alle covarianten Bildungen übereinstimmen, ist dann die Aufgabe, welche ich (im engeren Sinne, vgl. \$ 1) als das "Charakteristikenproblem algebraischer Formen" bezeichnen zu dürfen glaube, da sie in dem besonderen Falle, der hier zur Behandlung kommt, ebenso wie die an die Beweglichleit der Lösungen geknüpfte Problemstellung eine schärfere Fassung der von Chasles so bezeichneten Aufgabe vorstellt, und wirklich zu dem Resultate Chasles' hinführt.

Die Lösung dieser allgemeinen Aufgabe, für deren Behandlung die des Charakteristikenproblems der quadratischen Formen ungeachtet der einfachen Structur ihres Formensystems als Prototyp gelten kann, geschieht, wenn wir von besonderen Classen von Systemen von Formen absehen, die bei Formen von höherer als der zweiten Ordnung zu wesentlich verwickelteren Aufgaben führen, in zwei Schritten:

Bei dem ersten bandelt es sich um eine unmittelbare Verallgemeinerung des Bézout'schen Problems, von welcher jedoch immer nur besondere Fälle in Betracht kommen.

Der Inhalt der Fragestellungen dieser Art, deren Gesammtheit man vielleicht passend als das "Charakteristikenproblem der Punktmannichfaltigkeiten" bezeichnen könnte, lässt sich etwa so ausdrücken:

"Mehrere derselben algebraischen Mannichfaltigkeit angehörende Punktmannichfaltigkeiten, deren Dimensionen einander in geeigneter Weise ergänzen, schneiden sich im Allgemeinen in einer endlichen Zahl von Punkten. Wie gross ist diese Zahl? und wie viele Schnitt-

*) Es ist nämlich in den höheren Fällen kein Grund mehr vorhanden, warum man in dieser Hinsicht einzelne Covarianten - wie etwa diejenige, welche die Gleichnng einer Curve in Liniencoordinaten darstellt - vor den anderen auszeichnen soll. 
punkte werden absorbirt, wenu jene Mannichfaltigkeiten im besonderen Falle unendlich viele Punkte gemein haben?"

Erst bei dem zweiten Schritt tritt die Bedeutung in Kraft, welche man jenen Mannichfaltigkeiten, als von Systemen algebraischer Formen gebildet, beilegt: es kommt zu dem ersten Problem die der Theorie jener Systeme, als einem besonderen $\mathrm{Zweige} \mathrm{der} \mathrm{Algebra} \mathrm{eigenthüm-}$ liche Fragestellung hinzu:

"Wann eine gegebene Form mehreren Systemen wirklich, oder nar scheinbar zugleich angehört?"

Sind diese beiden Aufgaben in hinreichender Allgemeinheit erledigt, und damit auch das Charakteristikenproblem - was immer möglich sein muss, gleichviel, wie gross die Zabl der vorhandenen Invarianten ist -, so gesellt sich zu ihnen in besonderen Fällen ein drittes Problem: Die Frage nach der Gruppirung der erhaltenen Lösungen im einzelnen Falle. Hierher gehört dann vor Allem die Frage nach den Reductionen, welche die Beweglichkeit der Lösungen erleiden kann.

Bei Aufgaben der letzteren Art würde dann der ältere Begriff der "eigentlichen" Lösungen zur Geltung gelangen. Ich habe aber, um Missverständnissen vorzubeugen, dieses Wort ganz fallen lassen, spreche also nur von "scheinbaren" und "wirklichen" Lösungen, von welchen dann die letzteren weiterhin nach ihrer Beweglichkeit classificirt werden mögen.

Die hier gegebene Behandlung des Charakteristikenproblems der Kegelschnitte würde etwas kürzer ausgefallen sein, wenn wir uns, den Satz von Chasles als Ziel vor Augen, streng auf die Beibringung derjenigen Hilfsmittel beschränkt hätten, welche zur Erreichung desselben unbedingt nothwendig waren.

Es kann aber nicht der Zweck der Wissensehaft sein, irgend eine vereinzelte Wahrheit so kurz als möglich darzulegen: sie strebt vielmehr, ausgedehntere Gebiete, diese aber mit einer möglichst geringen Gesammtarbeit sich zu unterwerfen.

Daher sind bei der folgenden Untersuchung solche Sätze, welche nach meiner Meinung zu den elementaren Grundlagen einer jeden Theorie der Kegelschnittsysteme gehören, einer etwas ausführlicheren Behandlung unterzogen worden, als nöthig gewesen wäre, wenn dieselben als blosse Hilfssätze und Durchgangspunkte hätten dastehen sollen; so dass diese Darstellung, wie auch durch die Wahl des Titels angedeutet, vielleicht in der Hauptsache als eine auf die Geometrie der Kegelschnitte im Allgemeinen bezügliche Betrachtung anzusehen ist, von welcher eine Anwendung auf das Charakteristikenproblem gemacht wird. 
Was nun die bei einer Untersuchung dieser Art anzuwendenden Methoden anlangt, so schien mir die principielle und ausschliessliche Anwendung der Processe des sogenannten symbolischen Rechnens vor den anderen Verfahrungsarten der analytischen Geometrie den Vorzug zu verdienen.

Denn jene Methode allein genügt, richtig aufgefasst - was in dieser ohnehin schon ungewöhnlich umfangreichen Einleitung allerdings nicht auseinandergesetzt werden kann - m der gewiss berechtigten Forderung, zur Ableitung ihrer Wahrheiten keiner willkürlich eingeführten und daher überflüssigen Hilfsgrössen, wie Coordinaten sind, zu bedürfen*) - wiewohl eine flüchtige Betrachtung den entgegengesetzten Eindruck hervorbringen mag.

Indem dieselbe sich nur projectiver Processe bedient (deren jedem eine, aus der bisherigen Begründung dieser Theorie freilich nicht ersichtliche geometrische Bedeutung innewohnt), und daher viemals in die Lage hommt, die invarianie Natur ihrer Bildungen nachträglich beweisen zu müssen, vereinigt sie alleint, so lange sie aus diesem Kreise von Operationen grundsätzlich niemals heraustritt, den Hauptvorzug der synthetischen Geometrie - nämlich keinen überfliissigen Schritt zu thun - mit dem Vortheil der analytischen Methode - der Darstellung der geometrischen Gebilde durch Gleichungen; ja es lässt sich zeigen, dass ihr materieller Inhalt sich mit dem der synthetisehen Geometrie deckt, soweit man letztere auf linear ausfubrbare Construetionen beschränkt; wobei sie aber vor der Sehwesterdisciplin das voraus bat, dass sie allgemeine Methoden besitzt, deren Zulänglichkeit a priori bekannt ist, und dieselbe - was nicht gering anzuschlagen ist - durch die von vorn herein gegebene streng systematische Grup. pierung des Stoffes übertrifft.

Ausserdem aber - und dies allein wïrde hinreichen, die hohe Bedeutung jener, von Seiten der eigentlichen Geometer, wie ich glaube, nicht hivreichend gewürdigten Methode darzuthun - ist die principielle Anwendung jener invarianten Processe das einzige Mittel, welches die Gleichungen der geometrischen Gebilde in der denkbar allgemeinsten. und zugleich einfachsten Form darzustellen im Stande ist, da sie nicht mehr als das Nothwendige und Wesentliche zum Ausdruck bringt. Die gewöhnlichen Methoden körnnen eine äbnliche Einfachheit bloss uater Verzichtleistung auf Allgemeinheit erreichen, nämlich durch

*) H. Grassmann's grossartige Schöpfong ist zwar anch frei von Coordinatem, ist dafür aber von dem Begriffe des Maasses wesentlich abhängig, Ind daher für die Zwecke der projectiven Geometrie, wiewohl allen anderen Methoden, aussex der symbolischen, überlegen, dennoch nicht die geeignete Methode. Das eigentliche Gebiet für die Anwendungen der Ansdehnungslehre ist die Mechanik, in der sie ja auch ihren Ursprang genommen hat. 
Einführung specieller Coordinatensysteme, d. h. durch eimen sachlich ganz unbegründeten Kunstgriff, und auch das nur in sehr einfachen Fällen.

Was die Bezeichnung jener Processe betrifft, so habe ich mich der in Deutschland üblichen "symbolischen" Schreibart angeschlossen, weil diese am leichtesten $z u$ handhaben ist, wiewohl in theoretischer Hinsicht Cayley's, sowie besonders die aus Grassmann's Lückenausdrücken entspringende Bezeichnungsweise gewisse Vorzäge besitzen.

Eine Abweichung von der gebräuchlichen Darstellung habe ich mir nur insofern gestattet, als ich nicht, wie es gewöhnlich geschieht, Coordinaten etwa eines Punktes als Veränderliche ansehe, sondern im Sinne Grassmann's, diesen Punkt selbst; so dass, wenn in $\$ 3$ ein Punkt als „Parameter" einer Mannichfaltigkeit von fünf Dimensionen eingeführt wird, bei Anwendung der Coordinatenmethode die sechs homogenen Coordinaten dieses Punktes als eben so viele unabhängige (homogene) Parameter zu betrachten wären.

Die hier zu Grunde gelegte, in dieser Gestalt zuerst von Herrn Stroh (,Zur Theorie der Combinanten", Math. Annalen Bd. XXII S. 393 u. ff.) benutzte Parameterdarstellung linearer Mannichfaltigkeiten von Formen derselben Ordnung ist übrigens nicht, wie es vielleicht den Anschein hat, als ein willkürlicher Kunstgriff, sondern nur als der naturgemässe Ausdruck der Thatsache anzusehen, dass diese Formen in doppelter Hinsicht Gegenstand der Betrachtung werden: im vorliegenden Falle erscheint der-Kegelschnitt einmal als Ort von Punkten oder Linien in der Ebene - wobei also diese als veränderlich angesehen werden - das andere Mal als Element der ganzen, von allen Kegelschnitten der Ebene gebildeten Mannichfaltigkeit wobei der Kegelschnitt selbst veränderlich gedacht wird.

Man hat das hiermit berührte Verhältniss bisher so dargestellt, dass man die Coefficienten der Gleichung des Kegelschnittes (ich bleibe bei dem concreten Falle, wiewohl die Sache natürlich allgemeine Geltung hat) unmittelbar als homogene Coordinaten eines Punktes im senären Gebiete (Raum von fünf Dimensionen) deutete. Gegen ein solches Verfahren, einen so bedeutenden Fortschritt seine erste Einführung auch repräsentiren mochte, ist aber u. A. einznwenden, dass die rein projective Anffassung von Coefficienten einer Kegelschnittgleichung nichts weiss, dass bei seiner Anwendung die senär-invarianten Bildungen nicht, wie doch zu verlangen ist, auch formal als solche hervortreten, und dass es bei weitergehenden Untersuchungen bald genug die nöthige Einfachbeit vermissen lässt - wie es denn überhaupt derselben Kritik nnterliegt, wie der Gebrauch des Coordinatenrechnens im Allgemeinen.

Die doppelte Auffassung selbst aber ist bei Untersuchungen, wie 
die vorliegende, kaum zu umgehen. Denn die sogenannte „Abbildung auf die Punkte eimes höheren Raumes", deren einfachster analytischer Ausdruck jene Parameterdarstellung ist, dient nicht allein wesentlich zur klareren Auffassung bekannter Dinge* ${ }^{*}$, indem sie den wirklich vorhandenen Analogien mit Verhältnissen der gewöhnlichen Geometrie auch in der Form ihrer Sätze Rechnung trägt, sondern sie führt auch auf neue Wabrheiten, die zu entdecken auf anderem Wege sehr schwer gewesen sein würde, schon darum, weil ihre blosse Aussprache bei Verzichtleistung auf jene Analogie ausserordentlich verwickelt werden würde. ${ }^{* *}$ )

Es wäre daher, selbst auf dem Felde der rein synthetischen Methode, Pedanterie, wenn man aus metaphysischen Gründen, wie dem: „dass man sich höhere Räume nicht vorstellen könne, und mithin auch nicht von einer "Geometrie" (als etwas wesentlich auf der Anschauung Beruhendem) in solchen Räumen sprechen dürfe", auf solche Vortheile verzichten wollte, oder wenn man den an sich vollkommen berechtigten Satz dagegen in's Feld führen wollte, "dass man sich die Verhältnisse der ebenen Geometrie auch müsse klar machen können, ohne aus der Ebene hinauszutreten" - da ja ein solches Hinaustreten lediglich in der Ausdrucksweise stattfindet, während die begrifflichen Operationen, für die man unnöthiger Weise neue Namen erfinden müsste, wollte man jenen Analogien aus dem Wege gehen, dadurch natürlich nicht geändert werden, dass man „Punkt" statt „Kegelschnitt" sagt.

*) Wie mannichfache Abwege würden nicht in der Charakteristikentheorie vermieden worden sein, wenn man von ihr einen ausgiebigeren Gebranch bätte machen wollen!

**) Hierher gehören z. B. die meisten der Sätze, welche von der (hier mit $T$ bezeichneten) quadratischen Transformation handeln, die in der Geometrie der Kegelschnitte eine ansgezeichnete Stellung einnimmt.

Hinsichtlich der Kegelschnitte wurde die in Rede stehende Abbildung zuerst von Cayley betrachtet (, On the curves, which satisfy given conditions" Phil. Trans. 1868 vol. 158), der jedoch die Doppelnatur derselben nicht hervorhob. Die Kenntniss des Umstandes, dass mit jener Abbildung der Curven $\boldsymbol{n}^{\text {ter }}$ Ordnung zugleich eine Abbildung der Curven $\boldsymbol{n}^{\text {tor }}$ Classe verbunden sei, verdanken wir vorzïglich den Untersuehungen der Herren Rosanes und Reye (s. besonders die Darstellnng dieser Theorie bei H. Grassmann: „Verwendung der Ausdehnungslehre für die allgemeine Theorie der Polaren und den Zusammenhang algebraischer Gebilde" in Crelle's Journal Bd. 84, S. 273).

Neuerdings wurde dieselbe Abbildung der Kegelschnitte einer Ebene auf geometrischem Wege mit grosser Ausführlichkeit untersucht von Herrn Veronese (Le superficie omaloide ecc. Atti della R. A. dei Lincei, serie $3^{\mathrm{a}}$, vol, XIX, 1884) und fast gleichzeitig von Herrn Segre (,,Considerazioni intorno alla geometria delle coniche" ecc. Atti delle Scienze di Torino, vol. XX, 1885). Die geometrischen Resultate von $\$ 3$ und $\S 4$ der vorliegenden Arbeit sind in jenen zumeist bereits enthalten. 
Ich glaube sogar, nicht zu weit zu gehen, wenn ich in der Aufstellung und zwiefachen geometrischen Interpretation des vollständigen Formensystems der ternär-quadratischen, senär-linearen Form, welche jene Parameterdarstellung vermittelt, die Fundamentalaufgabe der Geometrie der Kegelschnitte erblicke.*)

Die Betrachtung derartiger Formen besitzt aber auch ein selbstständiges Interesse: Formen, die Veränderliche verschiedener von einander unabhängiger Gebiete enthalten, sind ebenso wie die Connexe Clebsch's als elementare Grundgebilde der Geometrie anzusehen; und zwar sind sie die allgemeinsten Objecte, welche den Methoden der Formentheorie unterworfen sind. Von geometrischer Seite haben sich einige derselben, besonders solche, welche mehrere Arten von Veränderlichen linear enthalten, und unter dem Namen der Systeme projectiver Erzeugungsarten von Curven oder Oberflächen bekannt sind, schon vor längerer Zeit der Betrachtung aufgedrängt, und sind neuerdings immer mehr in den Vordergrund getreten; auch lassen sich wohl eine Reihe von Untersuchungen, bei denen es weniger angenfällig ist, auf das Studium derartiger Formen zurückführen.

Die Algebra stellt dieselben von vorn herein als selbstständige Gebilde hin, welche, den Curven oder Flächen gleichwerthig, eine principielle Berücksichtigung verdienen, gleichgiltig ob sie zu bereits Bekanntem binleiten, oder nicht. Sie eröffnen der Geometrie ein noch kaum betretenes, überraschend ergiebiges Arbeitsfeld, welches ohne allzugrosse Mühe die merkwürdigsten Resultate ernten lässt.

Zum Theil wegen der veränderten Fassung des Charakteristikenproblems, zum Theil wegen der eben entwickelten methodischen Anschauungen des Verfassers hat von älteren, dem Gegenstande nach mit der vorliegenden zusammenhängenden Untersuchungen ausser einer eleganten, von Herrn Halphen eingeführten und von Herrn Schubert zu einem eigenthümlichen Calcül ausgebildeten symbolischen Schreibart für diese Betrachtung beinahe Nichts verwerthet werden können; es sind daher auch keine anderen Vorkenntnisse zum Studium derselben erforderlich, als die der Grundlagen der symbolischen Methode.**)

*) Die Bedeutung, welche dieser Form in der Theorie der Kegelschnitte zakommt, kann man am besten ans dem Umstande ermessen, dass man - wie hier beiläufig erwähnt werden mag - das volle. Formensystem beliebig vieler ternärer quadratischer Formen angeben kann, sobald man für diese Form ein endliches, vollständiges System aufgestellt hat. Aehnliches gilt natürlich von allen analogen Parameterdarstellangen.

*) Diese sind entwickelt in den folgenden Abhandlungen:

Clebsch ,Ueber symbolisehe Darstellung algebraischer Formen". Crelle's Journal Bd. 59, woselbst der Fundamentalsatz $x \alpha \tau^{\prime} \varepsilon \xi \chi^{\prime} \eta^{\prime} \nu$ dieser Theorie auf- 
Um dies zu ermöglichen, ist das Wenige, das aus älteren Darstellungen herübergenommen werden konnte, hier reproducirt worden. Es war im Wesentlichen nur die bereits von Chasles angegebene Zurückführung des auf mehr als zwei Kegelschnittsysteme bezüglichen Charakteristikenproblems auf die zwei Systeme betreffende Aufgabe.

1.

\section{Das Charakteristikenproblem.}

Wir gehen nun dazu über, die Aufgabe, um welche es sich handeln soll, genauer festzustellen, und zwar allgemein für beliebige algebraische Formen mit beliebig vielen Veränderlichen desselben Gebietes oder verschiedener Gebiete.

Wir nehmen an, es befinde sich in einem System algebraischer Formen, welches durch das Verschwinden gewisser Invarianten und Covarianten definirt ist, deren Gesammtheit wir mit $A$ bezeichnen wollen, eine Form, für welche gewisse andere covariante Bildungen $B$ verschwinden.

Dann wird die Gesammtheit der zu dem System (A) gehörigen Formen, für welche $B=0$ ist, eine Mannichfaltigkeit von niedrigerer Dimension bilden, als das - als irreducibel angenommene - System $(A)$ selbst, da sonst die Formen $B$ zu den Formen $A$ gehörten.

Es wird also in dem System $(A)$ unendlich viele, die gegebene Form enthaltende, eiufach ausgedehnte algebraische Mannichfaltigkeiten geben, für deren sämmtliche Formen keine der Covarianten $B$ oder höchstens eine einzelne zu $B$ gehörige Invariante $B_{1}$ verschwindet. Auf einer solchen Mannichfaltigkeit nun wird man immer einen Parameter $t$, welcher für die gegebene Form den Werth $t_{0}$ annimmt, so einführen können, dass jeder der Ausdrücke $B$ dividirt durch eine ganze Potenz von $\left(t-t_{0}\right)$ für den Werth $t=t_{0}$ gegen eine endliche

gestellt wird, nämlich der Satz, dass man durch die symbolischen Operationen alle rationalen Invarianten umbeschränkt veränderlicher Formen exhält;

Clebsch: ,Ueber eine Fundamentalaufgabe der Invariantentheorie", Gött. Abhdlg. Bd. 17, 1872, wo der Satz über die Zurüekführbarkeit von Formen mit mehreren Veränderlichen ("Reihen von Veränderlichen" in der Terminologie von Clebsch) auf die Formen ihres "eigentlich reducirten Systems" bewiesen wird, und

Gordan, „Ueber Combinanten", Math. Annalen Bd. 5, wo die nach den Formen des eigentlich reducirten Systems fortschreitenden Reihenentwicklungen, soweit sie hier in Betracht kommen, angegeben sind.

Ans der Theorie der ternären quadratischen Formen wird nur das vorausgesetzt, was etwa in Clebsch-Lindemann, "Vorlesungen über Geometrie" darüber gesagt ist. 
Grenze convergirt ( $B_{1}$ ausgenommen, das seinen Werth Null beibehält). Es wird dann in der Umgebung des Punktes $t_{0}$ jener Mannichfaltigkeit $B_{i}=\left(t-t_{0}\right)^{x_{i}} \cdot B_{i}^{\prime}$, wo $B_{i}^{\prime}$ für $t=t_{0}$ nicht mehr verschwindet.

Man erhält so für jede zu $B$ gehörige Invariante oder Covariante einen bis auf eine Potenz eines constanten Factors bestimmten Wertb, und für jede absolute Invariante und Covariante einen völlig bestimmten Werth*), wenn man jede Form $B_{i}$ durch die zugehörige $B_{i}^{\prime}$ ersetzt; und swar sind die $B_{i}^{\prime}$ mit der Grundform und unter einander durch dieselben identischen Relationen verknüpft, welche die $B_{i}$ verbanden, so lange sie nicht identisch Null waren**). - Ich bezeichne nun die gegebene Form, für welche noch mehr als eine Invariante oder Covarianten $B_{i}$ verschwindeu, die nicht zu $A$ gehören, als unvollständig in Bezug auf das System von Invarianten und Covarianten $A$; als in Bezug auf dieses System vollständig dagegen, wenn man alle $B_{i}$ durch die auf die angegebene Art bestimmten Formen $B_{i}^{\prime}$ ersetzt hat.

Als "vollständige Form" im engeren Sinne wollen wir dieselbe bezeichnen, wenn das System $A$ nur aus denjenigen Invarianten und Covarianten besteht, welche für alle Formen der gleichen Ordnung, Classe etc. identisch Null sind, wenn also die durch $A=0$ definirte Mannichfaltigkeit von Formen $(A)$ die höchstmögliche Dimension hat, und (folglich) linear ist.

Es werden nun jeder unvollständigen Form unendlich viele vollständige Formen zugehören, welche man durch die verschiedenen Grenzprocesse der geschilderten Art erhält. Haben wir insbesondere eine unvollständige Form $P$ als Glied einer algebraischen, zwei- oder mehrfach ausgedehnten Mannichfaltigkeit $M$ gegeben, welche der durch das Verschwinden von $A$ definirten Mannichfaltigkeit $(A)$ angehört, ohne zugleich einer Mannichfaltigkeit anzugehören, welche durch das Verschwinden eines $A$ umfassenden Systems ron Invarianten und Covarianten definirt ist, so wird man $P$ noch auf unendlich viele Arten mit Hilfe einfach ausgedehnter Mannichfaltigkeiten vervollständigen können, welche dem System $M$ angehören. Führt man dies auf alle wesentlich verschiedenen Arten aus, so erhält man ein System von zu $P$ gehörigen, in Bezug auf $A$ vollständigen Formen, welches alle solche Formen umfasst, die als Grenzlagen von Formen der Mannichfalltigkeit $\boldsymbol{M}$ angesehen werden können. Dieses System wird im Allgemeinen nicht sämmtliche zu $P$ gehörige, in Bezug auf $A$ vollständige

*) Hierher ist in diesem Zusammenhange auch der Werth $\infty$ zu rechnen, da man, am den bestimmten Werth 0 zn erhalten, in diesem Falle nur den reciproken Ausdrack zu betrachten brancht.

*) Das Festhaiten an diesen Relationen ist der Kern der Sache; von hier abs lässt sich auch die Verallgemeinerung der im Text eingeführten Begriffe auf Bimultane Systeme vollziehen. 
Formen umfassen. Wir betrachten nun allein diejenigen derselben, welche in diesem System vorkommen, als zu der Mannichfaltigkeit $\boldsymbol{M}$ gehörig; die anderen, welche nicht durch innerhalb $\boldsymbol{M}$ verlaufende Grenzprocesse erhalten werden können, wollen wir als dem System $M$ nur "scheinbar" angehörig bezeichnen.

Dann werden wir eine unvollständige Form $P$, welche mehreren in (A) enthaltenen Systemen $\boldsymbol{M}, \boldsymbol{M}^{\prime}, \boldsymbol{M}^{\prime \prime}$... angehört, als denselben nur scheinbar zugleich angehörig zu betrachten haben, wenn keine der zu $M$ und $P$ gehörigen vollständigen Formen zugleich eine vollständige Form von $\boldsymbol{M}^{\prime}, \boldsymbol{M}^{\prime \prime}, \ldots$ ist, oder genauer, wenn unter den zu $\boldsymbol{M}$ gehörigen vollständigen Formen $P_{M}$ sich keine findet, deren ergänzende Formen $B_{i}^{\prime}$ von den ergänzenden Formen je einer zu $M^{\prime}, M^{\prime \prime} \ldots$ gehörigen vollständigen Form $\boldsymbol{P}_{\boldsymbol{H}^{\prime \prime}}, \boldsymbol{P}_{\boldsymbol{M}^{\prime \prime}} \ldots$ sich nur um dieselben Potenzen je eines für $\boldsymbol{M}^{\prime}, \boldsymbol{M}^{\prime \prime}, \ldots$ constanten Factors unterscheiden. Alle zu $P$ gehörigen vollständigen Formen dagegen, bei welchen das Letztere eintritt, betrachten wir als den Systemen $\boldsymbol{M}, \boldsymbol{M}^{\prime}, \boldsymbol{M}^{\prime \prime} \ldots$ wirklich zugleich angehörig.

Hinsichtlich der Multiplicität dieses Angehörens bedarf es noch besonderer, sich in jedem Falle von selbst ergebender Bestimmungen. Setzen wir dieselben als ausgeführt voraus, so könuen wir die Aufgabe, um welche es sich hier bandelt, kurz so aussprechen:

Es sind mehrere Systeme von Formen, $\boldsymbol{M}, \boldsymbol{M}^{\prime}, \boldsymbol{M}^{\prime \prime} \ldots$ gegeben, für deren sämmtliche Formen. alle Invarianten und Covarianten eines Systems $A$ verschwinden, ohne dass für sämmtliche Formen eines dieser Systeme - abgesehen von einer einzigen etwa verschwindenden Invariante - noch irgend eine andere covariante Bildung identisch Null ist.

Anzugeben, wann eine diesen Systemen gemeinschaftlich angehörende unvollständige Form denselben scheinbar, oder wirklich zugleich angehört; und, falls die Zahl der allen Systemen gemeinsamen vollständigen Formen eine endliche ist, diese Zahl zu bestimmen.

Wir wollen nun diese Fragen unter der Voraussetzung, dass wir es mit vollständigen Formen im engeren Sinne zu thun haben, für die ternären quadratischen Formen in dem Falle behandeln, wo ein einfach - und ein vierfach ausgedehntes System zum Durchschnitt zu bringen sind.

\section{2.}

Das Charakteristikenproblem der ternären quadratischen Formen.

Die eben formulirte Aufgabe erfährt eine wesentliche Vereinfachung für die quadratischen Formen (und ähnlich für alle Formen, die nicht mehr als eine Invariante haben) durch den Umstand, dass eine solche Form im Allgemeinen nicht unvollständig sein kann; oder mit anderen Worten, dass eine quadratische Form, wenn eine covariante Bildang 
derselben verschwindet, die nicht für jede quadratische Form verschwindet, von weniger Constanten abhängt, als die allgemeinste quadratische Form*).

Wir behaupten insbesondere in Bezug auf die ternären quadratischen Formen, die uns bier allein angehen, dass die Covariante $(a b u)^{2}$ einer solehen Form $a_{x}{ }^{2 * s}$ ) identisch verschwinden muss, sobald irgend eine Covariante derselben identisch verschwindet, die nicht für jede ternäre quadratische Form Null ist.

In der That, nehmen wir an, es verschwinde irgend eine Covariante von $a_{x}{ }^{2}$ identisch. Dann verschwinden sämmtliche Formen ibres eigentlich redusirten Systemes ebenfalls identisch. Diese verschwinden nun entweder für jede Form $a_{x}{ }^{2}$, oder es muss, falls nicht die gegebene Form selbst den Factor $(a b c)^{2}$ haben soll, sich wenigstens eine Form unter denselben befinden, die von diesem Factor frei ist, und sich also auf Grund der von Herrn Gordan angegebenen Entwickelung als eine nach Potenzen von $u_{x}^{2} \cdot(a b c)^{2}$ fortschreitende Reihe darstellen lässt, in welcher der Coefficient der nullten Potenz ein Product einer Potenz von $a_{x}{ }^{2}$ und einer Potenz von $(a b u)^{2}$ ist.

Da auch diese Reihe nicht identisch verschwinden kann, ohne dass ihre einzelnen Glieder verschwinden, und $a_{x}{ }^{2}$ nicht verschwindet, so folgt $(a b u)^{2} \equiv 0$.

Da die Invariante $(a b c)^{2}$ Null in der zweiten Ordnung wird, wenn $(a b u)^{2}$ in der ersten Ordnung für beliebiges $u$ verschwindet, ergiebt sich zugleich, dass die Grenzlagen aller übrigen Covarianten bestimmt sind, sobald man die ron $(a b u)^{2}$ auf die im $\$ 1$ angegebene Art bestimmt hat; so dass man, um zu entscheiden, ob eine zwei Systemen angehörige unvollständige Form $a_{x}^{2}$ denselben scheinbar, oder wirklich zugleich angehört, nur darauf zu achten hat, ob unter den Formen, die man nach $\S 1$ an Stelle der Covariante $(a b u)^{2}$ erhält, sich solche befinden, die sich nur um einen Factor unterscheiden, oder nicht.

3.

Die doppelte Parameterdarstellung der Kegelschnitte einer Ebene.

Bei einer Untersuchung der Mannichfaltigkeit aller Kegelschnitte einer Ebene erscheint es, wie bereits hervorgehoben, naturgemäss,

*) Dies ist natürlich nur ein Aequivalent des Satzes, dass die irredncibelen quadratischen Formen linear in einander transformirbar sind; in Folge wovon man hier einen Unterschied zwischen allgemeinen und besonderen Formen machen kann, was in den höheren Fiallen nicht mehr angeht. Bei den Formen, welche nicht mehr als zoei Invarianten haben (insbesondere also auch den ternären cubischen und den binären biquadratischen Formen) findet noch eine ähnliche Vereinfachnng des Charakteristikenproblems statt. Jener Satz durfte in unserem Znsammenhange natürlich nicht benutat werden.

*) Ich bediene mich hier der allgemein üblichen Bezeichnungen. 
diese Gebilde auch formal als Elemente der von ihuen gebildeten Mannichfaltigkeit darzustellen, d. h. dieselben ron einem senären Parameter (oder genauer, einem Paar solcher Parameter, wie wir sogleich sehen werden) abbängig mu machen.

Wir werden uns also, behufs einer Untersuchnng zunächst der ron allen Curven zweiter Ordnung der Ebene gebildeten Maunichfaltigkeit einer Parameterdarstellung von der Form

$$
U_{\text {sf }} m_{x}^{2}=0
$$

bedienen. Hier verstehen wir unter $U_{X}=0, u_{x}=0$ (senäre Gebilde werden im Folgenden immer mit grossen, ternäre mit kleinen Bucl-staben bezeichnet) die (oincidenzbedingung eines Punktes $X$ und einer linearen Mannichfaltigkeit $U$ fünfter Stufe des senären, bezüglich eines Punktes $x$ und einer Geraden $u$ des ternären Gebietes, und es sollen, nach der gewöhnlichen Vorstellung "erst Symbole $\boldsymbol{M}$ mit Symbolen $m$ multiplicirt eine reelle Bedeutung erlangen".

Die Gleichung (1) wird im Allgemeinen - und wir betrachten hier nur den allgemeinen Fall, wiewohl die besonderen kein geringeres Interesse besitzen - sie wird im Allgemeinen zu je zwei verschiedenen Räumen $U$ zwei verschiedene Curven 2. $O$. liefern; und es wird, da linear abhängigen Räumen $U$ vermöge (1) ebenso beschaffene Curven entsprechen, auch ungekehrt möglich sein, zu einer gegebenen Curve 2. 0 . den ihr entsprechenden Raum $U$ eindeutig zu bestimmen.

Dasselbe gilt von der Abbildung der Mannichfaltigkeit aller Curven 2. Classe der Ebene auf die Punkte des senären Gebietes, welche, in der umgekehrten Richtung, ebenfalls durch (1) vermittelt wird.

Sei nämlich $u_{\alpha}{ }^{2}=0$ irgend eine Curve 2. Classe, so stellt

$$
U_{M} m_{\alpha}^{2}=0
$$

$U$ als veränderlich betrachtet, einen Punkt $X$ des senären Gebietes vor, und zwar entsprechen linear abhängigen Curven 2. Cl. linear abhängige Punkte. Es ist aber das Bestehen der Gleichnng (1 a) einerseits die Bedingung dafür, dass die Curve 2. 0 . deren Gleichung $U_{3} m_{x}{ }^{2}=0$ ist, und die Curre.2. C. $u_{\alpha}^{2}=0$ einander conjugirt sind; andererseits dafür, dass der Raum $V$ durch den $u_{\alpha}{ }^{2}$ entsprechenden Punkt $X$ hindurchgeht. Würden nun 6 linear unabhängigen Curven 2. Cl. nicht ebenso beschaffene Punkte entsprechen, sondern diese bereits in einem linearen Gebiet fünfter Stufe enthalten sein, so würde die letzterem entsprechende Curve 2. 0 . zu jenen 6 Curven 2. Classe conjugirt sein, was nicht angeht.

Also ist zugleich mit der durch (1) vermittelten ein-eindeutigen Abbildung der Räume $U$ des sesären Gebietes $R_{5}$ auf die Mannichfaltigkeit der Curven 2. 0 . der Ebene eine ebenfalls eindeutig umkehrbare Abbildung der Curven 2. Cl. auf die Punkte $X$ dieses Gebietes gegeben. Conjugirten Curven entsprechen incidente Elementenpaare von $R_{5}$. 
Die Gleichungen (1) und (1 a) geben uns zu jedem $R_{4}{ }^{1}: U$ von $R_{\mathrm{g}}$ die zugehörige Curve 2. 0 . und zu jeder Curve 2. Ol. den zugehörigen Punkt $X$ von $R_{5}$. Da wir wissen, dass die inversen Schritte ebenfalls ausführbar sind, so tritt nun die Aufgabe an uns heran, die Formeln, durch welche dies geschieht, und die, wie wir von vornberein sagen können, den Formeln (1) und (1 a) völlig gleichgebant sind und zu ihnen in umkehrbarer Beziehung stehen, wirklich herzustellen.

Wir baben zu diesem Behufe nach dem vorstehenden Satze nur mit Hilfe der Formel (1) die Curven 2. $O$. aufzusuchen, welche irgend fünf linear unabhängigen, einen gegebenen Punkt $X$ enthaltenden $R_{4}{ }^{1}$ von $R_{5}: V_{1}, U_{2} \ldots U_{5}$ entsprechen, und dann die Gleichung der zu ihnen gemeinsam conjugirten Curve 2. Cl. zu bilden, welche in Bezug auf $U_{1} \ldots U_{5}$ die Combinanteneigenschaft haben muss, dieselben also nur in der Verbindung:

$$
\left(U_{1} U_{2} \ldots U_{\overline{5}} U\right) \equiv U_{x}
$$

enthalten kann. In der That ist die ternäre Form 2. Cl.:

$$
U_{1_{M_{1}}} \ldots{J_{5}}_{M_{5}}\left\{\begin{array}{r}
\left(m_{1} m_{2} u\right)\left(m_{3} m_{4} u\right)\left(m_{1} m_{3} m_{5}\right)\left(m_{2} m_{4} m_{5}\right) \\
-\left(m_{1} m_{3} u\right)\left(m_{2} m_{4} u\right)\left(m_{1} m_{2} m_{5}\right)\left(m_{3} m_{4} m_{5}\right)
\end{array}\right\}
$$

welche, gleich Null gesetzt, jene Curve darstellt, da sie bei Vertauschung je zweier Symbole $\boldsymbol{M}_{\boldsymbol{x}} \boldsymbol{m}_{\boldsymbol{x}}{ }^{2}$ das Vorzeichen wechselt, identisch gleich:

$$
\begin{aligned}
& \frac{2}{5 !}\left(U_{1_{M_{4}}} \cdots U_{5_{H_{3}}}\right)\left(m_{1} m_{2} u\right)\left(m_{3} m_{4} u\right)\left(m_{1} m_{3} m_{5}\right)\left(m_{2} m_{4} m_{5}\right) \\
\equiv & \frac{2}{5 !}\left(M_{1} M_{2} \cdots M_{5} X\right)\left(m_{1} m_{2} u\right)\left(m_{3} m_{4} u\right)\left(m_{1} m_{3} m_{5}\right)\left(m_{2} m_{4} m_{5}\right)
\end{aligned}
$$

Dieser Ausdruck, für welchen wir kurz

$$
N_{X} u_{n}^{2}
$$

schreiben wollen, hat die Form der Gleichung (1). Er liefert, gleich Null gesetzt, die einem Punkte $X$ von $R_{5}$ entsprechende Curve 2. Classe; ebenso die Gleichung

$$
N_{X} a_{n}^{2}=0
$$

den einer gegebenen Curve 2. O.: $a_{x}{ }^{2}$ entsprechenden $R_{4}{ }^{1}$.

Sucht man mit Hilfe der Gleichung (2) zu einem Punkt $X$ die Gleichung der ihm entsprechenden Curve 2. Cl., und dann nach (1) zu dieser wieder den entsprechenden Punkt, so muss man zu dem Pankte $X$ zurückgelangen, und ebenso muss sich die Bedingung dafür, dass die einer gegebenen Curre 2. 0 . und einer gegebenen Curve 2. Cl. entsprechenden Gebilde incident sind, auf die Bedingung des Conjugirtsein's der ersteren reduciren. Dies findet seinen analytischen Ausdruck in den durah die Gordan'schen Reihenentwickelungen sich ergebenden Identitäten: 


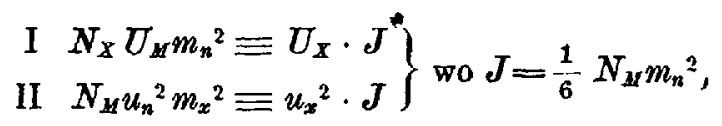

welche die Reduction jeder Covariante von (1) geben; die einen der symbolischen Factoren $N_{M}$ oder $m_{n}^{2}$ besitzt.

Das Verschwinden der (einzigen) Invariante $J$ der Form (1) wird durch die Bedingung ausgeschlossen, dass linear unabhängigen Räumen $R_{4}{ }^{1}$ eben so beschaffeue Curven 2. 0 . entsprechen sollen.

4.

Der Ort der ansgearteten Kegelschnitte.

Da eine ternäre Form 2. 0. zu ibrer Covariante 2. Cl. im Allgemeinen in umkehrbarer Beziehung steht, so ist sie bei der im vorigen Paragraphen betrachteten Abbildung zweimal vertreten: einmal durch einen Raum $R_{4}{ }^{1}$, den wir mit $W$ bezeichnen wollen, das andere mal durch einen Punkt $Z$. Beide sind, zufolge der Gleichungen (1) und (2) der eine aus dem anderen durch die Gleichungen zu bestimmen:

$$
\begin{aligned}
& W_{A^{2}} U_{A} \equiv W_{M} W_{M^{\prime}} U_{M^{\prime \prime}}\left(m m^{\prime} m^{\prime \prime}\right)^{2}=0 \text {, } \\
& L_{Z}^{2} L_{X} \equiv N_{Z} N_{Z}^{\prime} N_{X}^{\prime \prime}\left(\begin{array}{lll}
n & n^{\prime} & n^{\prime \prime}
\end{array}\right)^{2}=0 \text {. }
\end{aligned}
$$

Sucht man aus (3) den dem Ranme $W$ entsprechenden Punkt $Z$, uud dann aus (4) rückwärts den $Z$ entsprechenden Raum $R_{4}{ }^{1}$, so muss man, falls die Invariante des durch $W$ vorgestellten Kegelschnittes von Null verschieden ist, wieder zu dem Raum $W$ zurückgelautgen. In der That wird zu Folge der Reductionsformeln I und II:

III

IV

$$
\begin{aligned}
& L_{\perp} L_{\perp} U_{A^{2}} U_{A^{2}} L_{X} \equiv \frac{4}{3} U_{A}^{3} \cdot U_{X} \cdot J^{3} \\
& L_{\perp} L_{\perp} L_{X}^{2} L_{X}^{\prime 2} U_{\perp} \equiv \frac{4}{3} L_{X}^{3} \cdot U_{X} \cdot J^{3}
\end{aligned}
$$

Wir haben es also mit einer (im Allgemeinen) ein-eindeutigen, quadratischen Transformation zu thun; und zwar ist, wie der Anblick der Gleichungen (3) und (4) lehrt, die Beziehung zwischen dem Punkte $Z$ und dem ihm zugeordneten Raume $W$ eine derartige, dass $Z$ die lineare Polare des Raumes $W$ in Bezug auf die $R_{4}{ }^{1}$-Mannichfaltigkeit dritter Ordnung: $U_{\Lambda^{3}}=0$, oder kurz $\Lambda^{3}$, und gleichzeitig $W$ die lineare Polare von $Z$ in Bezug anf den Raum $R_{4}^{3}$ dritter Ordnung $L_{x^{3}}=0$, oder kurz $L^{3}$ ist. ${ }^{*}$ )

*) kin ähnliches Vorkommen bietet bereits die ternäre Geometrie in der Beziehung zwischen einem Punkt und seiner Harmonikale in Bezag anf ein festes Dreieck dar. Hier kann sowohl der Punkt als lineare Polare der Geraden in 
Wir wollen im Folgenden diese quadratische Transformation, die wir noch eingehender zu untersuchen haben, kurz als die „Transformation $T^{\prime 6}$ bezeichnen, und die in derselben einander entsprechenden Mannichfaltigkeiten von Punkten und Räumen $R_{4}^{1}$ als , ,System $S^{\star c}$ und „System $\Sigma$ " einander gegenüberstellen.

Die beiden Mannichfaltigkeiten $L^{3}$ und $\Lambda^{3}$ gehen durch $\infty^{5}$ collineare Transformationen in sich über, welche den $\infty^{8}$ collinearen Transformationen der Ebene entsprechen, und werden durch eben so viele dualistische Transformationen in einander übergeführt, welche den dualistischen Transformationen der Ebene zugeordnet sind.*)

In Folge des Vorhandenseins der Transformationen der letzteren Art wird man bei der Betrachtung der Mannichfaltigkeiten $L^{3}$ und $\Lambda^{3}$ willkürlich von einer derselben den Ausgang nehmen dürfen, indem das Princip der Dualität die entsprechenden Eigenschaften der anderen liefert. Wir wählen der leichteren Aussprache der aufzustellenden Sätze wegen den Raum $L^{3}$, dessen Punkte allen Curven 2. Classe der Ebene zugeordnet sind, welche in ein Paar von Strahlenbüscheln (d. h. in ein Punktepaar) ausarten.

Die Gleichung $L_{X}{ }^{3}=0$ wird, wie sich aus dieser Bemerkung ergiebt, und wie eine leichte Rechnung bestätigt, identisch erfüllt, wenn man für $X$ einen Punkt einsetzt, dessen Gleichung die Form:

$$
U_{\mathcal{M}} m_{\boldsymbol{x}} m_{y}=0
$$

hat. Betrachtet man $x$ und $y$ als veränderlich, und lässt beide Punkte die ganze Ebene durchlaufen, so erbält man in der Gleichung (5) eine Parámeterdarstellung des Raumes $L^{3}$.

Lässt man $x$ und $y$ zusammenfallen, so verwandelt sich (5) in die Parameterdarstellung einer Fläche

$$
U_{M} m_{x}^{2}=0
$$

deren Punkte den Punkten des ternären Gebietes ausnahmslos eindeutig zugeordnet sind.

$\mathrm{Da}$ die Gleichung (6) von (1) nicht verschieden ist, folgt, dass den Punkten der Schnittcurve dieser Fläche mit einem $R_{4}^{1}$ in der Ebene eine Curve 2. 0. entspricht. Da sich zwei solche in vier Punkten schneiden, ist sie von der vierten Ordnung; wir bezeichnen sie deshalb mit $F_{2}{ }^{4}$, und ihr dualistisches Gegenstück mit $\Phi_{2}{ }^{4}$.

Man erkennt sofort, dass den Punkten einer Geraden der Ebene

Bezng auf die von den Ecken des Dreiecks dargestellte Curve 3. Classe, als anch die Gerade als lineare Polare des Punktes in Bezug auf die von den Seiten des Dreiecks gebildete Curve 3. O. betrachtet werden.

*) Es kann das Vorliegende geradezu als eine auf diese Transformationsgruppe bezügliche Untersuchung angesehen werden. 
die Punkte eines Kegelschnittes jener Fläche zugeordnet sind, und dass überhaupt Curveu $n^{\text {ter }}$ Ordnung der Ebene und Curven $2 n^{\text {ter }}$ Ordnung von $F_{2}{ }^{4}$ einander entsprechen. Zugleich ergiebt sich, dass $\Lambda^{3}$ der Ort aller derjenigen Räume $R_{4}{ }^{\prime}$ ist, welche $F_{2}{ }^{4}$ in einer zerfallenden Curve 4. 0 . schneiden, $d . h$. in eivem einzigen Punkte berähren, und $\Phi_{2}{ }^{4}$ der Ort derjenigen, welche $F_{2}^{4}$ längs eines ganzen Kegelschnittes berühren.

Die lineare Polare eines Punktes $Z$ von $F_{2}{ }^{4}$ in Bezug auf $L^{3}$ wird unbestimmt, wie sich auch unmittelbar aus dem identischen Verschwinden der Covariante $\left(\alpha \alpha^{\prime} x\right)^{2}$ eines Doppelpunktes $\left(u_{\alpha}\right)^{2}$ ergiebt, so dass also $F_{2}{ }^{4}$ auch durch die äquivalenten Gleichungssysteme:

$$
L_{Z}{ }^{2} L_{X} \equiv 0 \quad \text { oder } \quad N_{Z} N_{Z}^{\prime}\left(n n^{\prime} x\right)^{2} \equiv 0
$$

definirt ist. Die Punkte von $\boldsymbol{F}_{2}^{4}$, und zwar nur diese, sind daher Doppelpunkte von $L^{3}$; es folgt hieraus, dass $L^{3}$ von sämmtlichen Sehnen von $F_{2}{ }^{4}$ beschrieben wird. Den Punkten solcher Sehnen von $F_{2}{ }^{4}$, welche $F_{2}{ }^{4}$ in zwei getrennten Punkten treffen, entsprechen bierbei die Punktepaare einer allgemeinen quadratischen Involution einer Geraden; den Tangenten von $F_{2}{ }^{4}$ die Punktepaare einer Involution, deren Doppelelemente zusanumengefallen sind.

Es folgt weiter, dass der Raum $L^{3}$ von zwei Schaaren von je $\infty^{2}$ Ebenen beschrieben werden kann, von welchen die erste aus allen Ebenen besteht, welche $F_{2}{ }^{4}$ in einem Kegelschnitte treffen, und die Punkte enthalten, welche den Punktepaaren einer Graden des ternären Gebietes entsprechen; während die der zweiten Tangentialebenen ${ }^{*}$ ) von $F_{2}{ }^{4}$ sind und solche Punkte tragen, welche Punltepaaren $x y$ der Ebene entsprechen, die einen festen Punkt $x$ enthalten. Die Parameterdarstellung der Ebenen dieser "zweiten Art" wird daher

$$
\left(\boldsymbol{M} \boldsymbol{M}^{\prime} \boldsymbol{M}^{\prime \prime} X \boldsymbol{X} Z\right)\left(m n^{\prime} m^{\prime \prime}\right) m_{x} m_{x}^{\prime} m_{x}^{\prime \prime}=0 \text {. }
$$

Durch einen Punkt $U_{M} m_{\xi} m_{\eta}=$ von $L^{3}$ gehen zwei solcher Ebenen, deren Parameter $\xi$ und $\eta$ sind; - umgekehrt schneiden sich zwei Ebenen zweiter Art immer in einem Punkt von $L^{3}$.

Der Anblick der Gleichung (5) zeigt, dass irgend zwei feste Ebenen dieser Schaar durch die Gesammtheit der übrigen collinear auf einander

*) D. h. Ebenen, welche alle durch einen gegebenen Punkt $x$ von $F_{2}^{4}$ gehenden Tangenten dieser Flische enthalten. Eine solche Ebene trifft $F_{2}{ }^{4}$ in vier unendlich benachbarten Punkten, als deren Verbindungslinien irgend 6 in ihr gelegene Tangenten von $\boldsymbol{F}_{\mathbf{4}}^{\mathbf{4}}$ angesehen werden können, die eine Involution bilden. Andere Ebenen als diese beiden Arten giebt es nicht auf $L^{3}$; auch enthält $L^{3}$ keine Gerade, die $F_{2}^{4}$ nur in einem einzigen Punkte trifft (wohl aber solche, die $F_{2}^{4}$ gar nicht treffen), wie überhanpt keine Curve, die mit $F_{2}^{4}$ eine ungerade Zahl von Punkten gemein bätte. (S. § 6.) 
bezogen sind, woraus sich eine einfache projective Erzeugungsart von $F_{2}{ }^{4}$ und $L^{3}$ ergiebt.

Durch einen Punkt von $L^{3}$, dessen Gleichung

$$
U_{m} m_{\xi} m_{\eta}=0
$$

ist, geht ferner eine Ebene der erster Art, welche den durch die Punkte $(\xi)$ und $(\eta)$ von $F_{2}{ }^{4}$ gehenden Kegelschnitt dieser Fläche enthält, und also den gegebenen Punkt mit den Beriihrungspunkten der beiden von ihm an $F_{2}{ }^{4}$ gehenden Tangenten verbindet. Setzt man

$$
(\xi \eta x) \equiv u_{x}
$$

so erhält man hieraus die Parameterdarstellung der Ebenen erster Art:

$$
\left(\boldsymbol{M} \boldsymbol{M}^{\prime} \boldsymbol{M}^{\prime \prime} X \boldsymbol{Z} Z\right)\left(m m^{\prime} u\right)\left(m m^{\prime \prime} u\right)\left(m^{\prime} m^{\prime \prime} u\right)=0 \text {. }
$$

Zwei Ebenen dieser Art treffen sich in einem Punkte von $F_{2}{ }^{4}$ und liegen in einem Raum $R_{4}{ }^{1}$ von $\wedge^{3}$; eine Ebene erster und eine Ebene zweiter Art treffen sich im Allgemeinen nicht, im besonderen Falle aber in allen Punkten einer Tangente von $F_{2}^{4}$.

In gleicher Weise, wie $L^{3}$ wird auch $\Lambda^{3}$ von zwei Arten von Ebenen (d. h. hier $R_{4}{ }^{1}$-Bündeln) beschrieben, und zwar ergiebt sich aus der oben aufgestellten Definition von $\Lambda^{3}$ und $\Phi_{2}{ }^{4}$, dass dies wieder die beiden eben betrachteten Schaaren von Ebenen sind, jedoch vertauscht, so dass die Ebenen erster und zweiter Art von $L^{3}$ als Ebenen zweiter, bezüglich erster Art von $\Lambda^{3}$ bezeichnet werden müssen ${ }^{*}$ ).

Da die Fläche $F_{2}{ }^{4}$ auf die Punkte des ternären Gebietes eindeutig umkehrbar bezogen ist, hindert uns nichts, dieselbe geradezu als Träger desselben aufzufassen. Wir werden dadurch in den Stand gesetzt, jede ternäre Construction so auszusprechen, dass sie im Gewande einer senären erseheint, und umgekehrt, was häufig zur Vereinfachung derselben beiträgt, und uns ausserdem neue Eigenschaften der Mannichfaltigkeiten $L^{3}$ und $F_{2}{ }^{4}$ kennen lehrt.

So ergeben sich beispielsweise alle die bekannten Sätze, welche von den Eigenschaften conjugirter Kegelschnittsysteme handeln, fast unmittelbar aus nnserer Abbildung. Wir wollen uns jedoch hier nicht damit aufhalten, diese ganze Theorie zur Ableitung zu bringen, sondern nur einige Sätze hier anfügen, die auf das Polarsystem einer Curve 2. Cl. $u_{\alpha}^{2}$ Bezug haben.

Wir hatten gesehen, dass im System $S$ der Ort aller Punkte $P$, welche Curven 2. Classe zugeordnet sind, die sich auf eine gegebene Curve 2. 0 . stützen, derjenige Raum $R_{4}{ }^{1}$ ist, welcher die gegebene Curve aus dem ternären Gebiete $F_{2}{ }^{4}$ ausschneidet.

*) Es ergiebt sich hier die Bemerknng, dass die Punkte von $\boldsymbol{F}_{2}{ }^{4}$ aus deu Ebenen erster Art von $L^{3}$ durch collineare $R_{3}{ }^{1}$-Bündel projicirt werden - was man als eine zweite projective Erzengangart von $\boldsymbol{F}_{2}{ }^{4}$ ansehen mag. 
Daraus ergibt sich unmittelbar, dass die au einer gegebenen Curve 2. Cl. $u_{\alpha}{ }^{2}$ conjugirten Geradenpaare aus dem ternären Gebiet $F_{2}{ }^{4}$ durch diejenigen Räume $R_{4}{ }^{1}$ von $\Lambda^{3}$ ausgeschnitten werden, welche den jener Curve 2. Cl. entsprechenden Punkt $P$ enthalten, und dass die durch $P$ gehenden Räume $R_{3}{ }^{1}$ die Fläche $F_{2}{ }^{4}$ in den Punkten eines Polvierecks von $u_{\alpha}^{2}$ treffen.

Man erhält also za einer gegebenen Geraden den Pol in Bezug auf $u_{\alpha}{ }^{2}$, wenn man die Ebene erster Art, welche ihren Repräsentanten auf $F_{2}{ }^{4}$ enthält, mit $P$ durch einen $R_{3}{ }^{1}$ verbindet, der dann den Pol auf $F_{2}^{4}$ ausschneidet.

Umgekehrt wird im System $S$ (und analog im System $\Sigma$ ) dasjenige Kegelschnittsystem, welches von allen Kegelschnitten gebildet wird, in Bezug auf welche ein gegebener Punkt und eine gegebene Gerade Pol und Polare sind, durch die Punkte eines linearen $R_{3}{ }^{1}$ dargestellt, welcher die Repräsentanten beider auf $E_{2}{ }^{4}$ ausschneidet; und das gleichfalls zu sich selbst dualistische System aller Kegelschnitte, die ein gegebenes Poldreieck haben, ist im Systeme $S$ den Punkten einer Ebene zugeordnet, welche die Repräsentanten der Ecken jenes Dreiecks auf $F_{2}^{4}$ verbindet.

Den Punkten eines $R_{3}{ }^{1}$ von $S$, welcher $F_{2}{ }^{4}$ in vier consecutiven Punkten (einer Ebene zweiter Art) trifft, entsprechen die Curven 2. $\mathrm{Cl}$. der Ebene, welche zwei feste Gerade berühren; dual entsprechen den $R_{4}{ }^{1}$ einer Selne von $F_{2}{ }^{4}$ die Curven 2. 0 ., welthe zwei gegebene Punkte enthalten.

Dies mag genügen, um die Eigenschaften des Ortes der ausgearteten Kegelschnitte wenigstens im Allgemeinen zu charakterisiren.

Wir wenden uns nun zur Betrachtung der durch die Gleichungen (3) und (4) vermittelten Transformation $T$.

\section{5.}

Das Unbestimmtwerden der Transformation $T$.

Die Formeln (3) und (III) des vorigen Paragraphen sagen aus, dass einem $R_{4}{ }^{1} W$ von $\Sigma$, welcher der Gleichung $W_{\lambda^{3}}=0$ genügt, in der Transformation $T$ ein Punkt von $S$ entspricht, welchem bei erneuter Anwendung von $T$ kein bestimmter $R_{4}^{1}$ von $\Sigma$ mehr zugeordnet ist. Es folgt hieraus (was auch unmittelbar einleuchtet), dass den Räumen von $\mathrm{A}^{3}$ in der Transformation $T$ Punkte von $F_{2}{ }^{4}$, den Punkten von $L^{3}$ Räume $R_{4}{ }^{1}$ von $\Phi_{2}{ }^{4}$ zugeordnet sind. Ond zwar entspricht, wie leicht zu sehen, je $\infty^{2}$ Räumen von $\Lambda^{3}$, die durch eine Ebene zweiter Art $\Pi$ vone $L^{3}$ gehen, in der quadratischen Transformation der Punkt $P$, in welchem diese Ebene $F_{2}{ }^{4}$ beriihrt. Umgekehrt entsprechen jedem Punkte $P$ von $F_{2}^{4}$ als Grenzlagen der 
den benachbarten Punkten von $P$ in der Transformation $T$ zugeordneten Räume $\infty^{2}$ Räume $R_{4}{ }^{1}$ von $\Lambda^{3}$, welche alle durch die Tangentialebene $\Pi$ von $F_{2}{ }^{4}$ in $P$ hindurchgeheu. Diese Unbestimmtheit der Transformation $T$ für die Punkte von $F_{2}{ }^{4}$ wird aufgehoben, wenn man sich einem Punkte $P$ dieser Fläche auf einer bestimmten, nicht ganz auf $F_{2}{ }^{4}$ verlaufenden Curve $P Q$ nähert, sobald man nur festsetzt, dass als der dem Punkte $P$ dieser Curve entsprechende $R_{4}{ }^{1}$ von $\Sigma$ die Grenzlage des einem Punkte $X$ derselben in $T$ entsprechenden $R_{4}{ }^{1}$ gelten soll, welche derselbe erreicht, wenn $X$ mit $P$ zusammenfällt.

Die Bestimmung dieser Grenzlage lässt sich besonders leicht bewerkstelligen, wenn $P Q$ eine Gerade ist. Man erbält dann nämlich als Gleichung der dem Punkte $P$ von $P Q$ entsprechenden Curve 2. O.:

$$
N_{P} N_{Q}^{\prime}\left(n n^{\prime} x\right)^{2}=0 \text {, }
$$

mithin als Gleichung des ihm in $\Sigma$ zugeordneten $R_{4}{ }^{1}$ :

$$
N_{P} N_{Q}^{\prime} N_{X}^{\prime \prime}\left(n n^{\prime} n^{\prime \prime}\right)^{2}=0 \text {. }
$$

Dieser Ausdruck ist aber eine Polare von

$$
K_{X}^{2} \equiv L_{P} L_{X}^{2} \equiv N_{P} N_{X}^{\prime} N_{X}^{\prime \prime}\left(n n^{\prime} n^{\prime \prime}\right)^{2} \text {. }
$$

Um zu einer durch einen Punkt $P$ von $F_{2}{ }^{4}$ gehenden Geraden den zugeordneten Raum $R_{4}{ }^{1}$ zu finden, betrachte man also den Tangentialkegel $K^{2}$ von $L^{3}$ im Punkte $P$, welcher das Umhüllungsgebilde der zu $\Phi_{2}{ }^{4}$ gehörigen quadratischen $R_{4}{ }^{1}$-Mannichfaltigkeit $x^{2}$ von $\Pi$ ist. Dieser Raum enthält, da die linke Seite von (7) und also auch von (8) identisch verschwindet, sobald $Q$ der Ebene $\Pi$ angehört, die Tangentialebene $\Pi$ des Punktes $\boldsymbol{P}$ von $\boldsymbol{F}_{2}{ }^{4}$ doppeltzählend ${ }^{*}$ ), und kann daher von einer quadratischen Schaar von $\infty^{1}$ Räumen $R_{3}{ }^{1}$ beschrieben werden, welehe die Ebene $\Pi$ enthalten und dieselbe mit den durch $\boldsymbol{P}$ gehenden Ebenen erster Art von $L^{3}$ verbinden ${ }^{* *}$ ).

Um nun in einem Punkte $P$ von $F_{2}{ }^{4}$, der einer Geraden $P Q$ angehört, den ihm in $\Sigma$ entsprechenden Raum $R_{4}{ }^{1}$ zu finden, haben wir gemäss der Formel (8) einfach die Polare eines beliebigen Punktes $Q$ dieser Geraden in Bezug auf $\mathcal{K}^{2} \mathrm{zu}$ bilden. Es haben aber alle Punkte $Q$, welche in einem and demselben durch $\Pi$ gehenden Raum $R_{3}{ }^{1}$ liegen, die nämliche Polare. Also:

„Damit einem Punkte $P$ von $F_{2}{ }^{4}$ als Punkt zweier verschiedener Geraden vermöge der Transformation $T$ ein und derselbe $R_{4}{ }^{1}$ von $\wedge^{3}$ entspreche, ist es nothwendig und ausreichend, dass beide Gerade mit

*) Die quadratische Polare eines nicht auf $\boldsymbol{H}_{2}{ }^{4}$ gelegenen Punktes von $\boldsymbol{L}^{3}$ enthält, wie sich hier ebenfalls ergiebt, eine Gerade doppelt zählend, die Polare jenes Punktes in Bezng anf den Kegelschnitt von $\boldsymbol{F}_{2}^{4}$, in dessen Ebene er liegt.

**) Der Schnitt von $L^{3}$ and $K^{2}$ ist also doppeltzăhlend der Kegel $R_{3}^{3}$, welcher die Pankte von $F_{q}^{*}$ ans $P$ projicirt. 
der Tangentialebene $\Pi$ von $F_{2}{ }^{4}$ im $P$ unite $P$ durch einen und denselben Raum $R_{3}{ }^{1}$ verbunden werden können. Der ihnen dann gemeinsam entsprechende $R_{4}{ }^{1}$ von $\Lambda^{3}$ ist die Polare eines Punktes jenes $R_{3}{ }^{1}$ in Bezug auf den Tangentialliegel $K^{2}$ von $L^{3}$ im Punkte $P$, oder in Bezug auf die Enveloppe von $K^{2}$, die in dem $R_{4}{ }^{1}$-Bündel erster Art $\Pi$ von $\Lambda^{3}$ enthaltene quadratische $R_{4}{ }^{1}$-Mannichfaltigheit $x^{2}$ von $\Phi_{2}{ }^{4} \cdot "$

Diese Construction, die vielleicht an Anschaulichkeit noch gewinnt, wenn man sie in's Dualistische überträgt, wird nur dann unausführbar, wenn eine der beiden Geraden in der Ebene $\Pi$ selbst liegt. Dann aber erhält man den dem Punlite $P$ einer solchen Geraden entsprechenden $R_{4}{ }^{1}$ von $\Phi_{2}{ }^{4}$ ohne Weiteres, indem man den Tangentialraum eines beliebigen Punktes $Q$ derselben an $L^{3}$ construirt.

Führt man den Grenzübergang zu dem Punkte $P$ von $F_{2}{ }^{4}$ nicht auf einer Geraden, sondern auf einer beliebigen Curve aus, so erhält man die der Tangente derselben im Punkte $P$ nach obigem Satze zugeordnete Grenzlage, ausgenommen den Fall, wo diese Gerade in $\Pi$ liegt, indem dann der einem Punkt $Q$ jener Curve, welcher zu $P$ benachbart ist, in Bezug auf $K^{2}$ zugeordnete $R_{4}{ }^{1}$ unbestimmt wird.

Es kann also jeder einen Punkt $P$ von $F_{2}{ }^{4}$ enthaltende Curvenzweig rïclsichtlich der Transformation $T$ dwrch seine Tangente in $P$ ersetzt werden, wenn derselbe $F_{2}{ }^{4}$ nicht in $P$ berïhrt.

In diesem Falle wird eine besondere Untersuchung nothwendig.

6.

Einfach and vierfach ausgedehnte Mannichfaltigkeiten, der

Transformation $T$ unterworfen. Die Charakteristiken derselben.

Wir sind durch die Untersuchung von $\S 3$ in den Stand gesetzt, zu jedem durch ein System invarianter Bedingungen $\Pi=0$, welche etwa eine Curve 2. Classe $u_{\alpha}{ }^{2}$ zu erfüllen hat, definirten Kegelsehnittsysteme die Gleichungen der beiden ihm in $S$ und $\Sigma$ entsprechenden Mannichfaltigkeiten anzugeben.

Da man bekanntlich jede Form von $\Pi$ aus einer ganzen Function der in verschiedenen Veränderlichen geschriebenen Formen:

$$
u_{\alpha} v_{\alpha}, a_{x} a_{y} \equiv(\alpha \beta x)(\alpha \beta y), a_{\alpha}^{2}, u_{x}
$$

durch Faltung mit zu $u_{\alpha}^{2}$ fremden Formen ableiten kann, so erhält man jene Gleichungen, indem man in jeder so geschriebenen Form von $\Pi$ die Symbole von $u_{a}{ }^{2}, a_{x}{ }^{2}$ das eine $\mathrm{Mal}$ durch Symbole von $N_{X} u_{n}^{2}$, bezüglich $N_{X} N_{X}^{\prime}\left(n n^{\prime} x\right)^{2}$, das andere Mal durch Symbole von $U_{M} U_{M^{\prime}}\left(m m^{\prime} u\right)^{2}$, bezüglich $U_{M} m_{x}^{2}$ ersetzt.

Ebenso kann man zu jeder in $R_{5}$ gegebenen algebraischen Punktoder $R_{4}{ }^{1}$-Mannichfaltigkeit sofort die Gleichungen des entsprechenden 
Kegelschnittsystems herstellen, wenn man für $X$ Symbole von $U_{M} m_{\alpha}{ }^{2}$ oder für $U$ Symbole von $N_{X} a_{n}^{2}$ einführt.

Ist eine algebraische Punktmannichfaltigkeit des Systems $S$ durch eine Gleichung für $X$ gegeben, etwa $A_{X}{ }^{2}=0$, so entspricht ihr vermöge der Transformation $T$ in $\Sigma$ eine Maunichfaltigkeit

$$
U_{A^{(1)}}^{2} \cdots{U_{\mathbb{A}^{(l)}}^{2}}_{A_{\mathcal{A}^{(1)}}} \cdots A_{\mathbb{A}^{(l)}}=0
$$

von doppelt so hoher Ordnung. Geht man durch erneute Anwendung von $T$ wieder zu dem System $S$ zurück, so erhält man in Folge der Identität (IV):

$$
\begin{aligned}
& L_{X}^{(1)} L_{X}^{(1)} \cdots L_{X}^{(n)} L_{X}^{(l)} \cdot L_{\mathcal{A}^{(1)}}^{(1)} L_{\mathcal{A}^{(1)}}^{\prime(1)} \cdots L_{\mathcal{A}^{(l)}}^{(0)} L_{\mathcal{A}^{(l)}}^{(())} \cdot A_{\mathcal{A}^{(1)}} \cdots A_{\mathcal{A}^{(l)}} \\
& \equiv\left[\frac{4}{3} \cdot J^{3}\right]^{l} \cdot\left[L_{x}^{3}\right]^{l} \cdot A_{x}^{l}
\end{aligned}
$$

Es scheidet sich also der Factor $L_{X}{ }^{3} l$-mal ab, was daher rührt, dass die transformirte Mannichfaltigkeit $\Phi_{2}{ }^{4} l$-mal enthält.

Dass aber in der Transformation $T$ Mannichfaltigkeiten von den Ordnungen $l$ und $2 l$ einander entsprechen, ist offenbar eine Besonderheit: als den allgemeinen Fall werden wir denjenigen anzusehen haben, wo $A_{x}=0$ bereits selbst mit der Eigenschaft behaftet ist, welehe bei dem transformirten Raum die Abscheidung des Factors $L_{x}{ }^{3}$ bei Anwendung der Transformation $T$ herbeiführt.

Um die allgemeinste Form der Gleichung eines Raumes $M_{4}{ }^{l}$, welcher $\lambda$ mal durch $F_{2}^{4}$ geht, zu finden, setze man für die Symbole von $u_{\alpha}{ }^{2}$ die Symbole einer sonst beliebigen, in einem binären Parameter $t$ linearen, und in den Linien des ternären Gebietes quadratischen Form $\sigma_{t} u_{s}{ }^{2}$, deren Covariante $\sigma_{t} \sigma_{t}^{\prime}\left(s s^{\prime} x\right)^{2}$ für $t=t_{o}$ identisch verschwindet. Nehmen wir nun an, dass die Gerade, welche der durch erstere Form dargestellten Kegelschnittschaar im System $S$ entspricht:

$$
\left(M M^{\prime} X Y Z T\right)\left(\sigma \sigma^{\prime}\right) m_{s}^{2} m_{s^{\prime}}^{2}=0
$$

nicht in dem $F_{2}^{4}$ angehörigen Punkte $U_{M} m_{s}^{2} \sigma_{t_{0}}=0$ den Raum $A_{X^{2}}=0$ berührt, und dieser Punkt keine weitere Besonderheit $A_{X}{ }^{l}=0$ gegenüber besitzt, so wird der Ausdruck $A_{X^{l}}$ für $t=t_{o}$ in der $\lambda^{\text {ten }}$ Ordnung verschwinden müssen. Nehmen wir ferner an, es sei $A_{X^{l}}$ in der zu Anfang dieses Paragraphen angegebenen Weise geschrieben. Enthalten dann diejenigen Terme von $A_{X}{ }^{3}$, welche von $a_{\alpha}^{2}$ frei sind - und solche muss es immer geben, wenn $A_{X}^{l}$ irreducibel sein soll $-\lambda_{1}$ Sýmbole von $a_{x}^{2}$, so wird $A_{x}{ }^{l}$ unter der obigen Voraussetzung von der Ordnung $\lambda_{1}$ Null werden, $d a$ alle anderen Terme in höherem Grade verschwinden. Es folgt also $\lambda_{1}=\lambda$, und wir erhalten als allgemeinste Gleichungsform eines Raumes von der Ordnung $l$, welcher $\lambda$ mal durch $F_{2}{ }^{4}$ geht, einen Ausdrack vom Typus: 


$$
N_{X^{(1)}} \ldots N_{X^{(l)} \Pi_{1}}\left[\left(u_{x}^{2}\right),\left(\left(n n^{\prime \prime} x\right)^{2}\right)\right]=0
$$

wo $\Pi_{1}$ eine simultane Invariante von $u_{n}^{2}$ und $\left(n n^{\prime} x\right)^{2}$ mit beliebigen anderen Formen ist, in welcher die von $\left(n n^{\prime} n^{\prime \prime}\right)^{2}$ freien Glieder in den Symbolen von $u_{n}{ }^{2}$ bis zum Grade $l-2 \lambda$, in den Symbolen von $\left(n n^{\prime} x\right)^{2}$ bis zum Grade $\lambda$ ansteigen, obne dass weitere Symbole von $u_{n}{ }^{2} z u$ Symbolen von $\left(n n^{\prime} x\right)^{2}$ zusammengezogen werden könnten.

Die dieser Gleichung in dem transformirten System $\Sigma_{1}$ entsprechende aber wird:

$$
U_{M(1)} \cdots U_{M^{\prime}(2 l-3 l)} \Pi_{1}\left[\left(\left(m m^{\prime} u\right)^{2}\right),\left(m_{x}^{2}\right)\right]=0,
$$

ein Ausdruck, der eine $R_{4}{ }^{2}$-Mannichfaltigkeit von der Ordnung $l^{\prime}=2 l-3 \lambda$ darstellt, welehe $\lambda^{\prime}=l-2 \lambda$ mal durch $\Phi_{2}{ }^{4}$ geht.

„Wenn eine Punkt-und eine $R_{4}{ }^{1}$-Mannichfaltigkeit je eines der Systeme $S$ und $\Sigma$, mit den Ordnungen $l$ und $l$, die bezüglich $\lambda$ und $\lambda^{\prime}$ mal die Gebilde $F_{2}{ }^{4}$ und $\Phi_{2}{ }^{4}$ enthalten, in der Transformation $T$ einander entsprechen, so bestehen zwischen den Zahlen $l, l^{\prime}-\lambda, \lambda^{\prime}$ die Gleichungen:

$$
\begin{array}{ll}
l^{\prime}=2 l-3 \lambda & l=2 l^{\prime}-3 \lambda^{\prime} \\
\lambda^{\prime}=l-2 \lambda & \lambda=l^{\prime}-2 \lambda^{\prime}
\end{array}
$$

oder aufgelöst:

$$
\begin{array}{ll}
\lambda=\frac{2 l-l^{\prime}}{3} & l=2 \lambda+\lambda^{\prime} \\
\lambda^{\prime}=\frac{2 l^{\prime}-l}{3} & l^{\prime}=2 \lambda^{\prime}+\lambda .
\end{array}
$$

Die Zahlen $\lambda$ und $\lambda^{\prime}$ bezeichnet man (im engeren Sime) als "Charakteristiken" des durch beide Mannichfaltigkeiten zusammen dargestellten Kegelschnittsystems.

Wir fügen an dieser Stelle noch die analogen Gleichungen an, welche sich auf in der Transformation $T$ einander entsprechende einfach ausgedehnte Mannichfaltigkeiten der Systeme $S$ und $\Sigma$ beziehen.

Wir bezeichnen mit $q$ die Ordnung einer Curve des Systems $S$, mit $\delta$ die Zahl der Punkte, in welchen sie die Fläche $F_{2}{ }^{4}$ trifft; und mit denselben Bucbstaben, aber gestrichelt, die dualistischen Charaktere, welche der jener Curve im System $\Sigma$ entsprechenden Mannichfaltigkeit zugehören.

Dann erhält man unmittelbar, wenn man bedenkt, dass einer linearen Punktmanniehfaltigkeit von $S$ in $\Sigma$ eine $\Phi_{2}{ }^{4}$ entbaltende quadratische $R_{4}{ }^{3}$-Mannichfaltigkeit (zufolge der Gleichung (3) $\$ 4$ ) zugeordnet ist, und umgekehrt, und dass die Zabl der beweglichen Schnittelemente mit den in $T$ einander entsprechenden einfach ausgedehnten Mannichfaltigkeiten beiderseits gleich sein muss, die Relationen

$$
\begin{aligned}
2 q-\delta & =q^{\prime} \\
q & =2 q^{\prime}-\delta^{\prime} .
\end{aligned}
$$


Die Zahlen $q$ und $q^{\prime}$ nennen wir wiederum, wie gebräuchlich, "Charakteristiken" der durch beide Mannichfaltigkeiten zusammen vorgestellten einfach ausgedehnten Kegelschnittschaar.

Es ist $q$ die Zahl der Kegelschnitte derselben, welche als Curven 2. Classe aufgefasst, auf einer gegebenen Curve 2. O. ruhen, oder eine gegebene Gerade berühren; $q^{\prime}$ die Zahl der Curven 2. O. der Schaar, welche eine gegebene Curve 2. Cl. stützen, oder durch einen gegebenen Punkt gehen.

Beiläufig ergiebt sich, dass jede auf $L^{3}$ verlaufende Curve die Fläche $F_{2}{ }^{4}$ in einer geraden $\mathrm{Zahl}$ von Punkten treffen muss, da im anderen Falle $\Phi_{2}{ }^{4}$ eine $R_{4}$ '-Schaar ungerader Ordnung tragen könnte, was unmöglich ist.

\section{7.}

Das Dnbestimmt-Werden der Transformation $T$ fïr Pankte vierfach ausgedehnter Mannichfaltigkeiten.

Wir gehen nun dazu über, zu untersuchen, wie die Umgebung eines Panktes $\boldsymbol{P}$ einer vierfach ausgedehnten Mannichfaltigkeit $\boldsymbol{M}_{4}$ durch die Transformation $\mathcal{T}$ aufgelöst wird, wenn derselbe in der Fläche $F_{2}^{4}$ liegt.

Bezeichnen wir die transformirte Mannichfaltigkeit durch $\boldsymbol{M}_{4}^{\prime}$, so werden wir sagen können, dass dem Punkte $P$ von $M_{4}$ Elemente von $\boldsymbol{M}_{4}^{\prime}$ entsprechen, welche der Tangentialebene $\Pi$ von $\boldsymbol{F}_{2}{ }^{4}$ im Punkte $P$ angehören; nicht aber wird auch umgekehrt jedem $R_{4}{ }^{1}$ von $\Pi$ als einem Element von $\boldsymbol{M}_{4}^{\prime}$ in der Transformation $T$ der Punkt $\boldsymbol{P}$ zugeordnet sein; nämlich im Allgemeinen solchen Räumen nicht, welche zugleich der Mannichfaltigkeit $\Phi_{2}{ }^{4}$ angehören (\$ 5).

Wir wollen nun zwischen den $\Pi$ angehörigen Elementen von $M_{4}^{\prime}$, welche durch Auflösung des Ponktes $\mathcal{P}$ entstehen, und denen, bei welchen dieses nicht der Fall ist, eine Unterscheidung treffen, und denjenigen Theil von $\mathbf{M I}_{4}^{\prime}$, welcher durch die Transformation $T$ aus der Umgebung des Punktes $\boldsymbol{P}$ von $\boldsymbol{M}_{4}$ entstanden ist, durch Einschliessung in eckige Klammer kenntlich machen, also durch das Zeichen $\left[M_{4}^{\prime}\right]$ darstellen.

Dann gibt über das Verhalten eines beliebigen Raumes $R_{4}{ }^{1}$ von $\Pi$ als eines Elementes von $\left[M_{4}\right]$ der folgende Satz Auskunft:

I. Zerfällt der Tangentialkegel einer Mannichfaltigkeit $\boldsymbol{M}_{4}$, welche $\lambda$ mal die Fläche $F_{2}{ }^{4}$ enthält, in einem Punkte $P$ dieser Fläche in zwei Theile $\left(\varepsilon_{1}\right)$ und $\left(\varepsilon_{2}\right)$ von den Ordnungen $\varepsilon_{1}$ und $\varepsilon_{2}$, von welchen der erste $\left(\varepsilon_{1}\right)$ von $\infty^{1}$ Räumen $R_{3}{ }^{1}$ beschrieben werden kann, die durch die Tangentialebene $\Pi$ von $F_{2}{ }^{4}$ im $P$ unkte $P$ gehen, während dies bei dem zueiten nicht der Fall ist; wo hingegen der Kegel $\left(\varepsilon_{2}\right) \eta$ einzelne Räume $R_{3}{ }^{1}$ trägt, welche durch $\Pi$ gehen (jeden mit der gehörigen Multiplicität gerech- 
net) - so ist der ganze $R_{4}{ }^{\prime}$-Bündel $\Pi$ in der transformirten Masnichfaltigkeit $\left[\boldsymbol{M}_{1}^{\prime}\right] \varepsilon_{1}+\varepsilon_{2}-\lambda$ mal enthalten; er wird von $\left[\boldsymbol{M}_{4}^{\prime}\right]$,ausserdem" in einer einfach ausgedehnten Schaar von der Gesammtordnung $\varepsilon_{1}$,geschnitten", $\left.{ }^{*}\right)$ welche der den Kegel $\left(\varepsilon_{1}\right)$ erfïllenden $R_{3}{ }^{1}$.Schaar in dem ( $\$ 5$ betrachteten) Polarsystem der zu $\Pi$ gehörigen $R_{4}{ }^{1}$-Schaar $x^{2}$ von $\Phi_{i}{ }^{4}$ entspricht, und trägt $\eta$ einzelne ,Knotenelemente $\left.{ }^{4 * *}\right)$ von $\left[M_{4}{ }^{\prime}\right]$, welche jenen $\eta$ einzelnen Räumen in demselben Polarsystem zugeordnet sind.

Der Beweis des ersten Theiles unseres Satzes ergibt sich unmittelbar, wenn wir durch einen festen Raum $A$ der Ebene $\Pi$, der weder $\Phi_{2}{ }^{4}$ angehört, noch gegenüber $\left[\boldsymbol{M}_{4}^{\prime}\right]$ eine ausgezeichnete Lage hat, einen $R_{4}{ }^{1}$ - Büschel legen. Die Zahl der beweglichen gemeinsamen Elemente dieses Büschels und der Mannichfaltigkeit $\boldsymbol{M}_{4}^{\prime}$ gibt dann, von der Ordnung $l^{\prime}$ von $M_{4}^{\prime}$ abgezogen, die Multiplicität von $A$ als eines Elementes von $\boldsymbol{M}_{4}^{\prime}$, oder, was in diesem Falle dasselbe ist, von $\left[\boldsymbol{M}_{4}^{\prime}\right]$.

Diese Zahl muss aber gleich sein der Zahl der beweglichen, im Allgemeinen nicht auf $F_{2}^{4}$ gelegenen Sehnittpunkte, in welehen ein durch $P$ und zwei nicht ausgezeichnete andere Punkte von $F_{2}{ }^{4}$ gehender Kegelschnitt die Mannichfaltigkeit $\boldsymbol{M}_{\boldsymbol{t}}$ trifft; also, wenn $l$ die Ordnung von $M_{4}$ ist, gleich

$$
2 l-2 \lambda-\left(\varepsilon_{1}+\varepsilon_{2}\right)=l^{\prime}-\left(\varepsilon_{1}+\varepsilon_{2}-\lambda\right) .
$$

Um den zweiten Theil des obigen Satzes zu beweisen, betrachten wir alle Räume $A$ eines $R_{4}{ }^{1}$-Büschels $a$, dessen sämmtliche Räume durch $\Pi$ gehen, verbinden dieselben mit einem nicht durch $\Pi$ gehenden $R_{4}{ }^{1}: B$ durch Büschel $A B$, und suchen zu bestimmen, wie oft ausser den $\left(\varepsilon_{1}+\varepsilon_{2}-\lambda\right)$ Schnitträumen mit $M_{4}^{\prime}$, welche für jede Lage des Büschels $A B$ in den Raum $A$ hineinfallen, unabhängig von der Lage von $B$ weitere Coincidenzelemente von $M_{4}^{\prime}$ mit diesem Büschel in feste Räume $A$ fallen. Wir haben behauptet, dass die Gesammtzahl der im Büschel $a$ enthaltenen Räume dieser Art $\varepsilon_{1}$ ist, wenn man jeden so oft in Anschlag bringt, als seine Multiplicität als Element von $\left[M_{4}^{\prime}\right]$ die Zahl $\left(\varepsilon_{1}+\varepsilon_{2}-\lambda\right)$ um eine Einheit übertrifft.

*) So wollen wir abkürzend sagen, wenn ein $\boldsymbol{R}_{4}{ }^{1}$, der einen Büschel der Ebene $\Pi$ durchläuft, als Element von $\left[\boldsymbol{M}_{4}^{\prime}\right]$ eine Gesammt-Multiplicitätserhöhung im Betrage $\varepsilon_{1}$ erfährt. Bei der Bestimmung dieser Zahl ist zu beachten, dass consecative Räume unter Umständen verschiedene Multiplicitätserhöhnngen erfahren können.

**) Dies soll heissen, dass die Gesammt-Mnltiplicitätserhöhung, welche einzelne Räume eines einzelnen $R_{4}{ }^{1}$-Büschels von $\Pi$ erfahren, wenn man dieses Büschel um einen festen $R_{4}{ }^{2}$ sich drehen lässt, $\eta$ beträgt. Ich habe beide Erläuterungen unter den Text gestellt, um in den Wortlaut des Satzes nicht allzuviele Erklärungen aufnehmen za müßsen. 
Jedem der Büschel $A B$ des Systems $\Sigma$ entspricht nun in $S$ ein Kegelschnitt, "der durch $P$ und zwei weitere Punkte von $F_{2}{ }^{4}$ geht. Derselbe zerfällt in eine Sehne von $F_{2}^{4}$, welche $P$ mit einem anderen Punkte dieser Fläche verbindet, und eine zweite Gerade, welche einen im Allgemeinen von $\boldsymbol{P}$ verschiedenen Punkt jèner Sehne mit einem dritten Punkte von $F_{2}{ }^{4}$ verbindet, sobald $\operatorname{der} \operatorname{Raum} A$ in die zu $\Phi_{2}{ }^{4}$ gehörige $R_{4}{ }^{1}$-Schaar $x^{2}$ von $\Pi$ fällt.

Suchen wir zuerst die Schnittpunkte zu bestimmen, welche diese letztere Sehne absorbirt.

Den Räumen von $x^{2}$ entsprechen in dem System $S$ alle Punkte des Kegels dritter Ordnung $R_{3}{ }^{3}$, welcher $F_{2}{ }^{4}$ ans dem Punkte $\boldsymbol{P}$ projicirt. Nehmen wir nun an, dass dieser Kegel $\delta$ mal auf $M_{4}$ enthalten ist, so folgt, dass in der transformirten Mannichfaltigkeit $M_{4}$ eine Multiplieitätserböhung der Räume von $x^{2}$ gegenüber den übrigen Räumen von $\Pi$ stattfindet, deren Betrag gleich

$$
\left(\lambda^{\prime}+\delta\right)-\left(\varepsilon_{1}+\varepsilon_{2}-\lambda\right)
$$

ist, wo $\lambda^{\prime}$ angibt, wie oft $M_{4}^{\prime}$ durch $\Phi_{2}^{4}$ geht. Denn die Zahl der beweglichen, im Allgemeinen weder auf jenem Kegel noch auf $F_{2}{ }^{4}$ gelegenen Schnittpunkte der Geraden, welche dem Büschel $B A$ in dem Falle eigentlich entspricht, wo $A$ zu $x^{2}$ gehört - die Zahl der Schnittpunkte dieser Geraden mit $M_{4}$ ist $l-\lambda-\delta$, eine Zahl, die nur dann eine Erniedrigung erfährt, wenn eine durch $P$ gehende Ebene erster Art öfter als $\delta \mathrm{mal}$ auf $\boldsymbol{M}_{4}$ enthalten ist. Dieser Ausdruck, von $l$ abgezogen, gibt aber die Multiplicität von $x^{2}, \mathrm{~d}$. b. eines nicht weiter ausgezeichneten Raumes von $\boldsymbol{x}^{2}$ als eines Elementes von $\boldsymbol{M}_{\boldsymbol{4}}^{\prime}$ gleich

$$
l-2 \lambda+\delta=\lambda^{\prime}+\delta \text {. }
$$

Die hiermit ausgeführte Bestimmung der Multiplicität von $x^{2}$ in $\boldsymbol{M}_{4}^{\prime}$ kommt jedoch für unseren nächsten Zweck nicht in Betracht; wir haben in dem Doppelten dieser Zahl gerade die für Räume $\mathcal{A}$ des Büschels $a$ eintretende Multiplicitätserhöhung bestimmt, welche von den 'Theilen der Mannichfaltigkeit $\boldsymbol{M}_{4}^{\prime}$ herrührt, die in der Transformation $T$ nicht der Umgebung des Punktes $P$ entsprechen, also wiewohl sie mit $\left[\boldsymbol{M}_{4}^{\prime}\right]$ zusammenhängen werden - nicht zu $\left[\boldsymbol{M}_{4}^{\prime}\right]$ gehören.

Dagegen werden die ausserdem stattfindenden Multiplicitätserhöhungen, gleichgiltig, ob sie für Elemente von $x^{2}$ eintreten (oder vielmehr solchen unendlich benachbart sind), oder nicht, zu $\left[\boldsymbol{M}_{4}^{\prime}\right] \mathrm{zu}$ rechnen sein, da dieselben nur daher rühren können, dass Schnittpunkte der durch $\boldsymbol{P}$ gehenden Kegelschnitte mit $M_{4}$ von dem Punkte $P$ selbst absorbirt werden.

Durchläuft nun der Raum $A$ den Büschel $a$, so durchläuft die Tangente des dem Büschel $[B A]$ des Systems $\Sigma$ im Systeme $S$ ent- 
sprechenden Kegelschnittes im Punkte $P$ einen ebenen Strahlenbüschel; es gibt also unter diesen Kegelschnitten im Ganzen $\varepsilon_{1}+\varepsilon_{2}$ Curven, welche $\boldsymbol{M}_{4}$ im Punkte $P$ berühren. Aendern wix nun die Lage ron $B$, so wird sich diese Kegelschnittschaar so ändern, dass die in $P$ gezogenen Tangenten je zweier Curven derselben, die zu dem nämlichen Raum $A$ gehören, mit $\pi$ durch einen und denselben Raum $R_{3}{ }^{1}$ verbunden werden können ( $(5)$. Hierbei werden aber von den $\varepsilon_{1}+\varepsilon_{2}$ Räumen $A$, welche bei der ersten Lage von $B$ Berührungskegelschnitten entsprachen, nur diejenigen imuer wieder Berührungskegelschnitten zugeordnet sein, welche im ersten Falle zu solchen Kegelschnitten gehörten, deren Tangenten ein durch $\Pi$ gehender $R_{3}{ }^{\prime}$ enthält, welcher ganz auf dem Tangentenkegel von $\boldsymbol{M}_{4}$ im Punkte $\boldsymbol{P}$ liegt. Für diese Räume $\boldsymbol{A}$ allein jst die 'Zahl der Schnittelemente eines Büschels $B A$ mit $M_{4}^{\prime}$, welche in $A$ fallen, immer grösser als $\left(\varepsilon_{1}+\varepsilon_{2}-\lambda\right)$, wie man auch $B$ wählen möge, und sie allein sind daher Räume höberer Multiplicität. Ihre Gesammtzahl wird zufolge unserer Voraussetzung für allgemeine Lage des Büschels $a$ gleich $\varepsilon_{1}$. Ebenso aber ergibt sich der dritte Theil unserer Behauptung. -

Der hiermit bewiesene Satz $I$ setzt nur die Charakteristik $\lambda$ als bekannt voraus; sein Inhalt ist ron dem Werthe der zweiten Charakteristik $\lambda^{\prime}$, oder der Ordnung $l$ der zu transformirenden Mannjchfaltigkeit $M_{4}$ unabhängig, wie auch von vorne herein zu erwarten war, da es sich nur um eine Transformation der in der Umgebung eines Punktes $P$ von $M_{4}$ gelegenen Punkte handelt.

Eine solche Unabbängigkeit findet nicht mehr statt, wenn es darauf ankommt, die Gesammtmultiplicität der Räume von $\Pi$ als Elemente der ganzen Maunichfaltigkeit $M_{4}^{\prime}$ zu bestimmen, indem jetzt der Schaar $x^{2}$ angehörige Räume hinzutreten, welche die Transformation $T$ in von $P$ verschiedene Punkte überführt.

Heben wir aus den Daten der vorstehenden Entwickelung das hierauf bezügliche heraus, so können wir den folgenden Satz aufstellen, der eine Frgänzung des Satzes $I$ enthält:

II. Sind bei einer Mannichfaltigkeit $M_{4}$ mit den Charakteristiken $\lambda$ und $\lambda^{\prime}$ die Voraussetzungen des Satzes $I$ erfüllt; ist ferner der die Fläche $F_{2}^{4}$ aus $P$ projicierende Kegel $\delta$ mal auf $M_{4}$ enthalten, und erfahren einzelne Ebenen dieses Kegels eine Multiplicitätserhöhung ats Elemente von $\boldsymbol{M}_{1}$, im Gesammtbetrag $\xi-$ so ist die quadratische Mannichfaltigkeit $\varkappa^{2}$ von $\Phi_{2}^{4}$, welche der Ebene erster Art $\Pi$ von $\Lambda^{3}$ angehört, $\lambda^{\prime}+\delta$ mal auf $M_{4}^{\prime}$ enthalten; und es finden, ausser der hierdurch etwa bedingten Multiplicitätserhöhung aller Elemente von $x^{2}$ gegenïber den übrigen, $\left(\varepsilon_{1}+\varepsilon_{2}-\lambda\right)$ mal auf $M_{4}^{\prime}$ enthaltenen Räumen von $\Pi$ noch für $\xi$ einzelne Elemente von $x^{2}$ Multiplicitätserhöhungen statt, welche jenen $\xi$ Ebenen erster Art von $L^{3}$ entsprechen. 
8.

\section{Der Chasles'sche Satz.}

Wie in $\S 2$ bewiesen, kann eine zwei verschiedenen Systemen von Curven 2. Classe gemeinsame Curve $u_{\alpha}{ }^{2}=0$ denselben nur dann scheinbar zugleich angehören, wenn ihre Covariante $(\alpha \beta x)^{2}$ identiseh verschwindet. Ist dies der Fall, so hängt die Entscheidung, ob sie beiden Systemen wirklich, oder scheinbar zugleich angehöre, von der Bestimmung der Grenzwerthe dieser Covariante ab. Für unsere Parameterdarstellung hat dies die Bedeutung, dass nur ein auf $F_{2}^{4}$ gelegener Punkt, oder ein zu $\Phi_{2}{ }^{4}$ gehöriger Raum $R_{4}{ }^{1}$ einen Kegelschnitt darstellen kann, welcher zwei Systemen scheinbar zugleich angehört; und dass weiter ein Punkt von $F_{2}{ }^{4}$ eine beiden Systemen wirklich gemeinsame Curve repräsentirt, so oft ihm als einem Schnittpunkt der jene Systeme in $S$ darstellenden Mannichfaltigkeiten auch ein gemeinsames Element der transformirten Mannichfaltigkeiten in $\Sigma$ entspricht.

Zu entscheiden, wann dies eintritt, hat aber nach dem Vorausgeschickten hinsichtlich der Zusammenstellung einer einfach ausgedehnten Mannichfaltigkeit $\boldsymbol{M}_{1}$ und einer vierfach ausgedehnter $\boldsymbol{M}_{\boldsymbol{4}}$ keine Schwierigkeit mehr.

Nehmen wir zuvörderst an, die $\delta$ Sehnittpunkte der Curve $q^{\text {ter }}$ Ordnung $M_{1}$ und der Fläche $F_{2}^{4}$ seien alle getrennt. Dann geht aus Satz $I$. des vorigen $\S$ unmittelbar hervor, dass von der Zahl der Schnittpunkte von $\boldsymbol{M}_{1}$ mit der Mannichfaltigleit $l^{\text {ter }}$ Ordnung $\boldsymbol{M}_{4}$, welche von einem beliebigen dieser Punkte $\boldsymbol{P}$ absorbirt werden, durch die Transformation $T$ immer $\lambda$ verloren gehen, wenn $\boldsymbol{M}_{4}$ die Fläche $F_{2}{ }^{4} \lambda$ mal enthält.

Denn möge zunächst die Curve $\boldsymbol{M}_{1}$ einfach durch $P$ hindurchgehen, obne mit $\boldsymbol{M}_{4}$ dort eine Berührung zu haben, so dass nur $\left(\varepsilon_{1}+\varepsilon_{2}\right)$ Schnittpunkte von $\boldsymbol{M}_{1}$ und $\boldsymbol{M}_{4}$ in $\boldsymbol{P}$ fallen. Dann entspricht derselben in dem Systeme $\Sigma$ eine $R_{4}{ }^{1}$-Mannichfaltigkeit, welche einen in Bezug auf $\left[\boldsymbol{M}_{4}^{\prime}\right]$ nicht ausgezeichneten Raum von $\Pi$ einfach enthält, dort aber nicht $\left(\varepsilon_{1}+\varepsilon_{2}\right)$, sondern nur $\left(\varepsilon_{1}+\varepsilon_{2}\right)-\lambda$ Elemente mit $\boldsymbol{M}_{4}^{\prime}$ gemein hat. Ebenso viele Schnittpunkte verschwinden aber, wenn $M_{1}$ und $M_{4}$ einander in $P$ in irgend einem Grade berühren; denn dies hat nur zur Folge, dass ebenso viele Schnitträume von $\boldsymbol{M}_{1}{ }^{\prime}$ und $\boldsymbol{M}_{4}^{\prime}$, die zuvor nicht auf $\Pi$ lagen, nun auf $\Pi$ zu liegen kommen, als die Zahl der in $P$ vereinigten Schnittpankte von $\boldsymbol{M}_{1}$ und $\boldsymbol{M}_{4}$ die Zahl $\left(\varepsilon_{1}+\varepsilon_{2}\right)$ übersteigt, theils in Folge dessen, dass nun $M_{1}^{\prime}$ durch Räume höherer Multiplicität von $\boldsymbol{M}_{4}^{\prime}$ geht, theils deshalb, weil nun Berühruugen von $\boldsymbol{M}_{1}$ und $\boldsymbol{M}_{4}^{\prime}$ dort stattfinden: in allen Fällen sind $\lambda$ cemeinsame Elemente verschwanden. 
Daran wird aber auch nichts geändert, wenn die $\delta$ vereinzelten Schnittpunkte von $M_{1}$ und $F_{2}{ }^{4}$ zum Theil consecutiv werden, oder in mehrfache Punkte ron $M_{1}$ zusammenrïcken; so dass man unter allen Umständen nur $l q-\lambda \delta$, oder wenn man nach $\S 6$ die Charakteristiken $\lambda^{\prime}$ und $q^{\prime}$ einführt, $l q^{\prime}+\lambda^{\prime} q$ Schnittpunkte erhält, welchen in der Transformation $T$ gemeinsame Elemente von $\boldsymbol{M}_{1}^{\prime}$ und $\boldsymbol{M}_{4}^{\prime}$ entsprechen; ein Resultat, das in der symmetrischen Form des Ausdrucks $\lambda q^{\prime}+\lambda^{\prime} q$ eine Bestätigung findet, da man natürlich von $\boldsymbol{M}_{1}^{\prime}$ und $\boldsymbol{M}_{4}^{\prime}$ ausgehend zu dem nämlichen Ergebniss gelangen muss.

Hiermit ist der Satz von Chasles bewiesen, und wir haben, um denselben in rein ternär-geometrischer Form aussprechen $z u$ können, nur noch die entsprechende Bedeutung der Charakteristiken $\lambda$ und $\lambda^{\prime}$ des vierfach ausgedehnten Kegelschnittsystems zu ermitteln.

Man erhält dieselbe nnmittelbar, wenn man im Satze $I$ des vorigen $\S . \varepsilon_{1}=\lambda$ und $\varepsilon_{2}=0$ setzt, was offenbar der Fall ist, der für die Punkte von $F_{2}{ }^{4}$ im Allgemeinen eintritt: es wird $\lambda$ gleich der Zahl der Kegelschnitte des vierfach ausgedehnten Systems, welche in einer Involution von Strahlenpaaren, als einer Kegelschnittschaar mit den Charakteristiken $q=0, q^{\prime}=1$ enthalten sind; wobei natürlich vorausgesetzt wird, dass diese Involution keine ausgezeichnete Lage gegen das gegebene Kegelschnittsystem besitzt. *)

Dem Chasles'schen Satze lässt sich hiernach der folgende Ausdruck geben:

„Wenn von den Kegelschnitten eines einfach ausgedehnten Systems $q$ eine gegebene Gerade berïhren, und $q^{\prime}$ durch einen gegebenen Punkt gehen; und wenn von den Curven eines vierfach ausgedehnten Kegelschnittsystems $\lambda$ in einer gegebenen Involution von Strahlenpaaren, und $\lambda^{\prime}$ in einer gegebenen Involution von Punltepaaren liegen - dann gibt es

$$
\lambda q^{\prime}+\lambda^{\prime} q
$$

beiden Systemen gemeinsame Kegelschnitte, oder, im besonderen Falle, deren unendlich viele."

9.

Der erweiterte Chasles'sehe Satz nnd die Halphen'sche Symbolik.

Hat man nicht ein vierfach ausgedehntes Kegelschnittsystem mit einer einfach ausgedehnten Kegelschnittreihe, sondern fünf vierfach ausgedehnte Systeme zum Durchschnitt zu bringen, so braucht man

*) Bei der wirklichen Bestimmung der Zahl $a$ auf diesem Wege ist natürlich zu beachten, dass unter den Doppellinien des vierfach auggedehnten Systems, welche etwa in eine Doppellinie der Involution fallen, diejenigen ond nor diejenigen mitzuzählen sind, welche aich auch aus den Strahlenpaaren der Involution durch Grenzübergang ergeben. 
nun nicht mehr neue Grenzbetrachtungen anzustellen, da es durch einen einfachen, schon von Chasles (C. R. t. 58 p. 297) angewandten, in unserem Zusammenhange zulässigen Schluss gelingt, diesen Fall auf den eben behandelten zurückzufähren.

Wir bedienen uns, indem wir die Chasles'sche Betrachtung an dieser Stelle reproduciren, einer zuerst in ähnlichem Sinne von Herrn Hal phen (Comptes Rendus t. 76 p. 1074 oder Bulletin de la Société Matbém. de France t. 1. Année 1872-73 p. 236) gebrauchten Schreibart, derselben, die Herr Schubert seinem "Kalkül der abzählenden Geometrie" zu Grunde gelegt hat, und bezeichnen demnach die Zahl der Curven, in welchen sich mehrere in den Dimensionen geeignet gewählte Kegelschnittsysteme schneiden, durch das Product der Symbole, die wir für diese Systeme gebrauchen; wir bezeichnen also, wenn $L_{1}, L_{2}, \ldots L_{5}$ fünf beliebige einfach ausgedehnte Kegelschnitte sind, durch das Produkt $L_{1} L_{2} \ldots L_{5}$ die Zahl der ihnen wirklich gemeinsam angehörenden Kegelschnitte; insbesondere, wenn $\mu$ und $\nu$ vierfach ausgedehnte Systeme sind, welche in $\Sigma$, bezüglich in $S$ durch lineare Mannichfaltigkeiten repräsentirt werden, durch $\mu^{5}$ die Zahl der Curven, in welchen sich fünf verschiedene Systeme von der Art $\mu$ durchdringen u. s. w.

Die fünf Systemen $\mu$ oder $\nu$ gemeinsamen Curven kann man sofort angeben. Ihre Anzahlen sind die folgenden:

I.

$$
\mu^{5}=1, \quad \mu^{4} \nu=2 ; \quad \mu^{3} v^{2}=4 ; \quad \mu^{2} v^{3}=4 ; \quad \mu \nu^{4}=2 ; \quad \nu^{5}=1 .
$$

Aus diesen Zahlen kann man aber leicht mit Hilfe des Chaslesschen Satzes die Zahl der Curven finden, in welchen sich ein beliebig gegebenes vierfach ausgedehntes Kegelschnittsystem $L$ und vier Systeme $\mu, \nu$ durchdringen; man hat nur zu berücksichtigen, dass die Charakteristiken $q, q^{\prime}$. eines einfach ausgedehnten Systemes $Q$ als die Zahlen

$$
q=Q \nu \quad q^{\prime}=Q \mu
$$

definirt sind. Setat man hier für $Q$ der Reihe nach: $\mu^{4}, \mu^{3} \nu, \mu^{2} \nu^{2}$, $\mu \nu^{3}, \nu^{4}$, so erhält man die Tabelle:

II.

$$
\begin{aligned}
& L \mu^{4}=\lambda+2 \lambda^{\prime} \\
& L \mu^{3} \nu=2 \lambda+4 \lambda^{\prime} \\
& L \mu^{3} \nu^{2}=4 \lambda+4 \lambda^{\prime} \\
& L \mu \nu^{3}=4 \lambda+2 \lambda^{\prime} \\
& L \nu^{4}=2 \lambda+\lambda^{\prime},
\end{aligned}
$$

woraus die ebenfalls sehon von Chasles angegebenen Relationen:

$$
\begin{gathered}
L\left(2 \mu^{4}-\mu^{3} v\right)=0 \quad L\left(2 \nu^{4}-\mu \nu^{3}\right)=0 \\
L\left(4 \mu^{4}+4 \nu^{4}-3 \mu^{2} \nu^{2}\right)=0 .
\end{gathered}
$$


Aus diesen Ausdrücken,erbält man durch Wiederholung des obigen Schlusses die Charakteristiken der einfach ausgedehnten Systeme $L \mu^{3}$, $L \mu^{2} v \ldots$, und durch erneute Anwendung des Chasles'schen Satzes die Tabelle

III.

$$
\begin{aligned}
& L_{1} L_{2} \mu^{3}=\lambda_{1} \lambda_{2}+2\left(\lambda_{1} \lambda_{2}^{\prime}+\lambda_{1}^{\prime} \lambda_{2}\right)+4 \lambda_{1}^{\prime} \lambda_{2}^{\prime} \\
& L_{1} L_{2} \mu^{2} \nu=2 \lambda_{1} \lambda_{2}+4\left(\lambda_{1} \lambda_{2}^{\prime}+\lambda_{1}^{\prime} \lambda_{2}\right)+4 \lambda_{1}^{\prime} \lambda_{2}^{\prime} \\
& L_{1} L_{2} \mu \nu^{2}=4 \lambda_{1} \lambda_{2}+4\left(\lambda_{1} \lambda_{2}^{\prime}+\lambda_{1}^{\prime} \lambda_{2}\right)+2 \lambda_{1}^{\prime} \lambda_{2}^{\prime} \\
& L_{1} L_{2} \nu^{3}=4 \lambda_{1} \lambda_{2}+2\left(\lambda_{1} \lambda_{2}^{\prime}+\lambda_{1}^{\prime} \lambda_{2}\right)+\lambda_{1}^{\prime} \lambda_{2}^{\prime},
\end{aligned}
$$

woraus die bekannte Identität

$$
L_{1} L_{2}\left(2 \mu^{3}-3 \mu^{2} \nu+3 \mu \nu^{2}-2 \nu^{3}\right)=0
$$

folgt. So fortfahrend erhält man ferner die Tafeln

IV.

$$
\begin{gathered}
L_{1} L_{2} L_{3} \mu^{2}=\lambda_{1} \lambda_{2} \lambda_{3}+2 \Sigma \lambda_{1} \lambda_{2} \lambda_{3}^{\prime}+4 \Sigma \lambda_{1} \lambda_{2}^{\prime} \lambda_{3}^{\prime}+4 \lambda_{1}^{\prime} \lambda_{2}^{\prime} \lambda_{3}^{\prime} \\
L_{1} L_{2} L_{3} \mu \nu=2 \lambda_{1} \lambda_{2} \lambda_{3}+4 \Sigma \lambda_{1} \lambda_{2} \lambda_{3}^{\prime}+4 \Sigma \lambda_{1} \lambda_{2}^{\prime} \lambda_{3}^{\prime}+2 \lambda_{1}^{\prime} \lambda_{2}^{\prime} \lambda_{3}^{\prime} \\
L_{1} L_{2} L_{3} \nu^{2}=4 \lambda_{1} \lambda_{2} \lambda_{3}+4 \Sigma \lambda_{1} \lambda_{2} \lambda_{3}^{\prime}+2 \Sigma \lambda_{1} \lambda_{2}^{\prime} \lambda_{3}^{\prime}+\lambda_{1}^{\prime} \lambda_{2}^{\prime} \lambda_{3}^{\prime} \\
V . \\
L_{1} L_{2} L_{3} L_{4} \mu=\lambda_{1} \lambda_{2} \lambda_{3} \lambda_{4}+2 \Sigma \lambda_{1} \lambda_{2} \lambda_{3} \lambda_{4}^{\prime}+4 \Sigma \lambda_{1} \lambda_{2} \lambda_{3}^{\prime} \lambda_{4}^{\prime} \\
+4 \Sigma \lambda_{1} \lambda_{2}^{\prime} \lambda_{3}^{\prime} \lambda_{4}^{\prime}+2 \lambda_{1}^{\prime} \lambda_{2} \lambda_{3}^{\prime} \lambda_{4}^{\prime} \\
L_{1} L_{2} L_{3} L_{4} \nu=2 \lambda_{1} \lambda_{2} \lambda_{3} \lambda_{4}+4 \Sigma \lambda_{1} \lambda_{2} \lambda_{3} \lambda_{4}^{\prime}+4 \Sigma \lambda_{1} \lambda_{2} \lambda_{3}^{\prime} \lambda_{4}^{\prime} \\
+2 \Sigma \lambda_{1} \lambda_{2}^{\prime} \lambda_{3}^{\prime} \lambda_{4}^{\prime}+\lambda_{1}^{\prime} \lambda_{2}^{\prime} \lambda_{3}^{\prime} \lambda_{4}^{\prime}
\end{gathered}
$$

VI.

$$
\begin{aligned}
L_{1} L_{2} L_{3} L_{4} L_{5} & =\lambda_{1} \lambda_{2} \lambda_{3} \lambda_{4} \lambda_{5}+2 \Sigma \lambda_{1} \lambda_{2} \lambda_{3} \lambda_{4} \lambda_{5}^{\prime}+4 \Sigma \lambda_{1} \lambda_{2} \lambda_{3} \lambda_{4}^{\prime} \lambda_{5}^{\prime} \\
& +4 \Sigma \lambda_{1} \lambda_{2} \lambda_{3}{ }^{\prime} \lambda_{4}^{\prime} \lambda_{5}^{\prime}+2 \Sigma \lambda_{1} \lambda_{2}^{\prime} \lambda_{3}^{\prime} \lambda_{4}^{\prime} \lambda_{5}^{\prime}+\lambda_{1}^{\prime} \lambda_{2}^{\prime} \lambda_{3} \lambda_{4}^{\prime} \lambda_{5}^{\prime}
\end{aligned}
$$

Die Schlussformel, die alle früheren einschliesst, ist der gesuchte Ausdruck, welcher die Zahl der fünf beliebigen Kegelschnittsystemen gemeinsamen Curven angiebt. Er liefert z. B. die Zahl der Kegelschnitte einer Ebene, welche irgend fünf gegebene Curven berühren, wenn man $\lambda_{i}$ die Classe, $\lambda_{i}^{\prime}$ die Ordnung der $i^{\text {ten }}$ Curve bedeuten lässt - wie sich sofort aus der im vorigen $\$$ angegebenen Bestimmungsart der Charakteristiken $\lambda$ und $\lambda^{\prime}$ ergiebt.

Dem letzten Ausdruck hat Herr $\mathrm{Halphen}$ eine bemerkenswerthe symbolische Form gegeben, deren Nothwendigkeit wir nunmehr von vornherein einsehen können. Man kann nämlich, nachdem diese Formel einmal auf algebraischem Wege abgeleitet ist, die Bemerkung machen, dass dieselbe, da sie nur von den Charaliteristiken der einzelnen Systeme abhängt, dem Princip der „Erhaltung der An$z a h l^{\prime \prime}$ unterworfen ist, $d . h$. dass sie denselben Werth, wie zuvor an- 
gibt, wenn man an Stelle der gegebenen Systeme beliebige andere einführt, sobald diese nur die nämlichen Charakteristiken haben.

Wählt man insbesondere reducibele Systeme, so dass das gegebene System $L_{i}$ durch die Systeme $L_{i}^{\prime}, L_{i}^{\prime \prime} \ldots$ zusammengenommen vertreten wird, so erbält man nach dem Vorstehenden die Zahl der den Systemen $L_{1} \ldots L_{5}$ gemeinsamen Curven, wern man in Bezug auf jedes der Systeme $L_{i}, L_{k}, \ldots$ auf alle möglichen Arten je eines der dieselben vertretenden Theilsysteme $L_{i}^{(m)}, L_{k}^{(n)} \ldots$ herausgreift, die Zahl der diesen Gruppen gemeinsam angehörigen Curven bestimmt, und von allen diesen Zahlen die Summe bildet. Dies wird aber nur anders ausgedrückt, wenn man sagt: „Man bilde für jedes System die „symbolische" Summe

$$
L_{i}^{\prime}+L_{i}^{\prime \prime}+L_{i}^{\prime \prime}+\ldots \ldots
$$

multiplicire alle so entstandenen Summen mit einander, und ersetze nachher die Producte $L_{1}{ }^{(m)} L_{2}^{\left(m^{\prime}\right)} \ldots L_{5}^{(n)}$ durch die Zahl der Curven, welche die Systeme $L_{1}^{(m)}, L_{2}{ }^{\left(m^{\prime}\right)} \ldots L_{3}^{(n)}$ mit einander gemein haben."

Die einfachste Art, wie man ein System $L$ durch elementare Systeme mit denselben Charakteristiken $\lambda$ und $\lambda^{\prime}$ ersetzen kann, ist nun offenbar die, dass man $\lambda$ Systeme $\mu$ und $\lambda^{\prime}$ Systeme $\nu$ benutat. Wir setzen also, mit Rücksicht auf Fragestellungen der vorliegenden Art:

$$
L=\lambda \mu+\lambda^{\prime} \nu
$$

ein Ausdruck, den wir mit Herrn Halphen als den „Modul" des Systems $L$ bezeichnen.

Führt man diesen Ausdruck ein, so erhält man statt der Formel VI die folgende:

$$
L_{1} L_{2} L_{3} L_{4} L_{5}=\prod_{1}^{5}\left(\lambda_{i} \mu+\lambda_{i}^{\prime} \nu\right)
$$

eine Formel, die, wenn man sie ausrechnet, d. h. wenn man die Producte der $\mu$ und $\nu$ durch die in Tafel I. angegebenen Zahlen ersetzt, wieder in den Ausdruck VI übergeht.

Ebenso kann man aber auch jedes einfach ausgedehnte System durch einen "Modul" ersetzen, d. b. durch ein einfachstes zerfallendes System, welches dieselben Charakteristiken hat: man kann unmittelbar

$$
Q=q \cdot \sigma+q^{\prime} \cdot \tau
$$

schreiben, wo $\sigma$ eine Involution von Punktepaaren einer Geraden, $\tau$ eine Involution von Strahlenpaaren eines Punktes ist, und erhält dann den Satz von Chasles ausgedrückt durch die Formel:

$$
L Q=\left(\lambda \mu+\lambda^{\prime} \nu\right)\left(q \sigma+q^{\prime} \tau\right)
$$

wo bei der Ausrechnung

$2 \pi$ setzen ist.

$$
\mu \sigma=0 \quad \nu \tau=0 \quad \mu \tau=1 \quad \nu \sigma=1
$$


10.

\section{Die Beweglichkeit der Lösungen. Beispiel.}

Die Fälle, in welchen die Beweglichkeit der Lösungen des Charakteristikenproblems eine Reduction erfahren kann, lassen sich mit Hilfe der oben eingeführten Parameterdarstellung leicht übersehen.

Beschränken wir uns auf den wichtigsten Fall, wo im Systeme $S$ eine Curve $M_{1}$ mit einer vierfach ausgedehnten Mannichfaltigkeit $M_{4}$ zum Durchschnitt zu bringen ist.

Dann ist ohne Weiteres einlenchtend, dass ein der Fläche $F_{2}{ }^{4}$ nicht benachbarter Punkt von $\boldsymbol{M}_{1}$, welcher auf $\boldsymbol{M}_{4}$ liegt, eine beweglịche Lösung des Charakteristikenproblems darstellen muss, da man jeden Punkt des senären Gebietes der nicht auf $L^{3}$ liegt, und jeden Punkt von $L^{3}$, der nicht zu $F_{2}{ }^{4}$ benachbart ist, noch auf unendlich viele Arten durch eine der Collineationen, die $L^{3}$ in, sich transformiren, in jeden anderen Punkt gleicher Art überführen kann. Ebenso kann anch jeder Schnittpunkt, welcher einem Punkte $P$ von $F_{2}{ }^{4}$ benachbart ist, aber weder auf $F_{2}{ }^{4}$ noch auf $L^{3}$ liegt, durch eine lineare Transformation beseitigt werden, da nicht alle Fortschreitungsrichtungen, die von Punkten von $F_{2}^{4}$ ausgehen, zu $\boldsymbol{M}_{4}$ gehören können; desgleichen lassen sich alle wirklichen Lösungen entfernen, die dureh Punkte von $F_{2}{ }^{4}$ absorbirt werden, welche als Elemente von $M_{4}$ eine die Zahl $\lambda$ übersteigende Multiplicität besitzen. Derselbe Sehluss kann ferner gemacht werden, wenn die Curve $M_{1}$ in einem Punkte $P$ von $F_{2}{ }^{4}$ den Raum $L^{3}$ berïhrt, d. h. wenn sie, die etwa in $P *$ Punkte mit $F_{2}^{4}$ gemein bat, $L^{3}$ dort in mehr als $2 \varkappa$ Punkten trifft, $M_{4}$ aber den Raum $L^{3}$ nicht zugleich längs der ganzen Fläche $F_{2}^{4}$ berührt.

Tritt aber dieser letztere Umstand ein, enthält also der Taugentialkegel von $M_{4}$ in einem beliebigen Punkte von $F_{2}^{4}$ den Tangentialkegel von $L^{3}$ immer als Theil, und berührt gleichzeitig die Curre $M_{1}$ den Ranm $L^{3}$ in einem Punkte von $F_{2}{ }^{4}$, so ist wenigstens einer der daher rührenden Schnittpunkte von $\boldsymbol{M}_{1}$ und $\boldsymbol{M}_{\boldsymbol{1}}$ auf keine Weise durch lineare Transformation $\mathrm{zu}$ entiernen, da natürlich bei jeder Transformation der Gruppe, welche $L^{3}$ in sich überführt, die Tangentialkegel von $L^{3}$ in den Punkten von $F_{2}^{4}$ in einander übergehen.

In diesem Falle, und in diesem allein, wird also eine Verringerung der Beweglichkeit der Lösungen eintreten.

Einer Geraden des Systems $S$, welche $L^{3}$ in einem Punkte von $F_{2}{ }^{4}$ berührt, entspricht aber im System $\Sigma$ ein $R_{4}{ }^{1}$-Büschel, welcher mit $\Lambda^{3}$ in einem Raume von $\Phi_{2}^{4}$ einen Contact hat*), und beiden zu-

*) Allgemeiner entspricht einem Curvenzweig, welcher in einem Punkte $\boldsymbol{P}$ mit $F_{2}^{4} x$ Punkte, und mit $L^{3}$ ausser den hierdurch absorbirten $2 x$ Schnittpunkten noch $\boldsymbol{x}^{\prime}$ weitere gemein hat, im System $\Sigma$ eine $\boldsymbol{B}_{4}{ }^{1}$-Schaar, welche mit $\boldsymbol{\Phi}_{2}{ }^{4}$ 
sammen entspricht im ternären Gebiet ein Büschel, oder, was in diesem Falle dasselbe ist, eine Schaar von einander vierpanktig berührenden Kegelschnitten, welche die dritte Kegelschnittausartung enthält, die als Curve 2. Ordnung aufgefasst aus einer doppelt zählenden Geraden, und als Curve 2. Classe aus einem doppelt zählenden, auf jener Geraden enthaltenen Punkt besteht. Wir können daher das letzte Resultat geometrisch so ausdrücken :

„Die Lösungen des Charakteristikenproblems sind dann und nur dann alle beweglich, wenn entweder das einfach ausgedehnte System keinen, oder das vierfach ausgedehnte System nicht jeden der $\infty^{3}$ ausgearteten Kegelschnitte der dritten Art enthält."

In den anderen Fällen treten in dem einfach ausgedehnten System unbewegliche Lösungen auf, deren $\mathrm{Zahl}$ in jedem einzelnen Falle durch eine besondere Untersuchung bestimmt werden muss, die z. B. so ausgeführt werden kańn, wie es Herr $\mathrm{Halphen}$ angegeben hat.

Es hat dieses Auftreten unbeweglicher Curven für die wirkliche Auffindung der Lösungen des Charakteristikenproblems dieselben Erleichterungen im Gefolge, welche überhaupt in der Algebra durch den Umstand herbeigeführt werden, dass man von einer aufzulösenden Gleichung von vorne herein weiss, dass dieselbe mit einer anderen Gleichung eine gewisse Anzahl gemeinschaftlicher Wurzeln besitzt. -

Wir wollen nun zum Schluss die bisher in abstracto behandelten Verhältnisse an einem Beispiele näher ausführen, und die Gleichungen wirklich aufstellen, von deren Aufösung die Auffindung der einigen einfach gebauten Kegelschnittsystemen wirklich gemeinsamen Curven abhängt.

Als vierfach ausgedehntes Kegelschnittsystem nehmen wir dasjenige, dessen Curven mit zwei fest gegebenen Punkten (oder allgemeiner: Curven 2. Classe) $x$ und $y$ eine absolute Invariante haben, welcbe za der absoluten Invariante desselben Kegelschnittes mit zwei gegeben vorliegenden Geraden $u$ und $v$ (die man auch durch Curven 2. Ordnung ersetzen kann) in einem constanten Verhältnisse steht; wir betrachten also, wenn $a_{x}{ }^{2}=0$ die Punktgleichung, $u_{\alpha}^{2}=0$ die Liniengleichung eines und desselben Kegelschnittes ist, so dass

$$
a_{\alpha} a_{x} u_{\alpha}-\frac{1}{3} u_{x} \cdot a_{\alpha}^{2} \equiv 0,
$$

das Kegelschnittsystem, dessen Gleichung:

$$
\frac{\left(a_{x} a_{y}\right)^{2}}{a_{x}^{2} \cdot a_{y y^{2}}{ }^{2}}=c \frac{\left(u_{\alpha} v_{\alpha}\right)^{2}}{u_{\alpha}^{2} \cdot v_{\alpha}^{2}}
$$

$x^{\prime}$ Ränme, und mit $\Lambda^{3} x$ diesen consecntive Räume gemein hat. Die Zahlen $x^{\prime}$ and $x$ decken sich mit den Begriffen der "Ordnong" und "Classe" des ausgearteten Kegelschnittes bei Halphen. 
oder, nach Beseitigung des Nenners:

$$
\left(a_{x} a_{y}\right)^{2} \cdot u_{\alpha}^{2} \cdot v_{\alpha}^{2}-c \cdot a_{x}^{2} \cdot a_{y}^{2} \cdot\left(u_{\alpha} v_{\alpha}\right)^{2}=0
$$

ist, also ein Kegelschnittsystem mit den Charakteristiken $\lambda=\lambda^{\prime}=2$.

Dasselbe gehört im Allgemeinen nicht zu denjenigen, welche jeden ausgearteten Kegelschnitt der dritten Art enthalten, denn die Gleichung (1) wird im Allgemeinen nicht identisch erfüllt, wenn man darin

$$
a_{x}^{2}=(\xi \eta x)^{2} \quad u_{\alpha}^{2}=u \xi^{2}
$$

setzt. Dies tritt aber ein, sobald $c=1$, und wir haken daher in der Gleichung

$$
\left(a_{x} a_{y}\right)^{2} \cdot u_{\alpha}^{2} v_{x}^{2}-a_{x}^{2} \cdot a_{y}^{2} \cdot\left(u_{\alpha} v_{\alpha}\right)^{2}=0,
$$

welche den besouderen Fall vorstellt, wo die absoluten Invarianten geradezu gleich sind, ein Beispiel eines jener ausgezeichneten Kegelschnittsysteme, und zwar offenbar das einfachste von allen, da $\lambda$ und $\lambda^{\prime}$ für diese Systeme wenigstens den Werth 2 haben muss.*)

Diese Systeme nun wollen wir mit einem Kegelschnittbüschel und einigen ausgezeichneten Ausartungen desselben zum Durchschnitt bringen.

Ein Kegelschnittbüschel wìrd dargestellt dorch eine Gleichung von der Form:

$$
\alpha_{t} a_{x}^{2}=0
$$

wo $t$ ein binärer Parameter ist, und, nach der gewöhnlichen Ausdrucksweise, erst Symbole $\alpha$ mit Symbolen a multiplicirt reelle Bedeutung erlangen. Die cubische Gleichung, von welcher die Linienpaare des Büschels abhängen, ist

$$
A_{t}^{3}=\alpha_{t} \alpha_{t}^{\prime} \alpha_{t}^{\prime \prime}\left(a a^{\prime} a^{\prime \prime}\right)^{2}=0
$$

und hat die Discriminante $R=\left(\Delta \Delta^{\prime}\right)^{2}$, wenn wir mit $\Delta_{\imath}{ }^{2}$ die Hesse'sche Covariante $\left(A A^{\prime}\right)^{2} A_{t} A_{t}^{i}$ von $A_{t}{ }^{3}$ bezeichnen.

$R$ ist im allgemeinen Falle von Null verschieden, und von der Liniengleichung des Kegelschnittes (3): $\alpha_{t} \alpha_{i}^{\prime}\left(a a^{\prime} w\right)^{2}$ sondert sich kein binär-linearer Factor ab: man erhält durch Substitution der Form (3) in die Gleichung (1) [oder (2)] eine irreducibele Gleichung $6^{\text {ten }}$ Grades für $t$ :

$$
\begin{gathered}
\left(\alpha_{t} a_{x} a_{y}\right)^{2} \cdot \alpha_{t} \alpha_{t}^{\prime}\left(a a^{\prime} u\right)^{2} \cdot \alpha_{t} \alpha_{t}^{\prime}\left(a a^{\prime} v\right)^{2} \\
-c \cdot \alpha_{t} a_{x}{ }^{2} \cdot \alpha_{t} \alpha_{y}{ }^{2} \cdot\left[\alpha_{t} \alpha_{t}^{\prime}\left(a a^{\prime} u\right)\left(a a^{\prime} v\right)\right]^{2}=0
\end{gathered}
$$

entsprechend dem Chasles'schen Satze, da die Charakteristiken des allgemeinen Kegelschnittbüschels $q=2, q^{\prime}=1$ sind.

*) Es ist dieses Beispiel ron dem von Herrn Halphen angegebenen nicht wesentlich verschieden. 
Bei einem Büschel von Kegelschnitten dagegen, welcher eine doppelt zählende Gerade enthält und zugleich eine Kegelschnittschaar ist, haben wir $q=1, q^{\prime}=1$; wir können nach dem Chasles'schen Satze nur 4 wirkliche Lösungen erhalten, und es muss sich daher von jener Gleichung $6^{\text {ten }}$ Grades ein quadratischer Factor abscheiden (der die nun auftretenden scheinbaren Lösungen angiebt).

In der That haben wir in diesem Falle $R=0$ anzunehmen, es wird $\Delta_{t}^{2}$ ein Quadrat, gleich $\left(\Delta_{t}\right)^{2}$, und von der Liniengleichung des Kegelschnittbüschels muss sich der Factor $\Delta_{t}$ absondern, so dass wir

$$
\alpha_{t} \alpha_{t}^{\prime}\left(a a^{\prime} u\right)^{2} \equiv \Delta_{t} \cdot \beta_{t} u_{b}^{2}
$$

setzen können. $(\Delta \alpha) a_{x}{ }^{2}=0$ wird nun die Gleichung der Doppellinie des Büschels, $(\Delta \beta) u_{b}{ }^{2}=0$ die Gleichung des Punktepaares, welches jener Doppellinie als Curve 2. Classe zugeordnet ist.

Führen wir diese Voraussetzungen in die Gleichung (4) ein, so erhalten wir

$$
\left(\Delta_{t}\right)^{2}\left[\left(\alpha_{t} a_{x} a_{y}\right)^{2} \beta_{t} u_{b}^{2} \cdot \beta_{t} v_{b}^{2}-c \cdot \alpha_{t} a_{x}{ }^{2} \cdot \alpha_{t} a_{y}{ }^{2}\left(\beta_{t} u_{b} v_{b}\right)^{2}\right]=0,
$$

es scheidet sich also in der That der Factor $\left(\Delta_{t}\right)^{2} a b$, und es bleibt nur noch eine Gleichung $4^{\text {ten }}$ Grades zur Bestimmung der beiden Systemen wirklich gemeinsamen Curven aufzulösen.

Ein analoges Ergebniss erhaiten wir, wenn wir weiterhin die Annahme einführen, dass wir einen Büschel einander vierpunktig berührender Curven vor uns haben. Es wird dann $\Delta_{t}{ }^{2} \equiv 0, A_{t}{ }^{3}$ eine dritte Poten $z,=\left(\mathrm{A}_{t}\right)^{3}$, und es ist $\alpha_{t} \alpha_{t}^{\prime}\left(a a^{\prime} u\right)^{2} \equiv \mathrm{A}_{t} \cdot \gamma_{t} u_{c}{ }^{2} \mathrm{zu}$ setzen. Es scheidet sich jetzt der Factor $A_{t}{ }^{2} a b$, und wir erhalten wiederum eine Gleichung vierten Grades. Von dieser können wir aber jetzt, in dem Falle, wo $c=1$, eine Wurzel von vorn herein angeben, denn es ist nun die Gleichung der Curve 2. Classe der Schaar: $(A \gamma) u_{c}{ }^{2}=0$, welche der Doppellinie $(A \alpha) a_{x}{ }^{2}=0$ des Büschels (1) entspricht, ein Quadrat geworden, $A$ ist also eine Wurzel der Gleichung:

$$
\left(\alpha_{t} a_{x} a_{y}\right)^{2} \gamma_{t} u_{c}^{2} \cdot \gamma_{t} v_{c}^{2}-\alpha_{t} a_{x}^{2} \cdot \alpha_{t} a_{y}^{2}\left(\gamma_{t} u_{c} v_{c}\right)^{2}=0
$$

und es bleibt nur noch eine Gleichung dritten Grades aufzulösen, welche allein die beweglichen Schnittcurven beider Systeme angiebt.

Sei endlich der Kegelschnittbüschel in eine Involution von Strahlenpaaren ausgeartet. Dann wird auch $A_{t}^{3} \equiv 0$, und es ist

$$
\alpha_{t} \alpha_{t}^{\prime}\left(a a^{\prime} u\right)^{2} \equiv C_{t}^{2} \cdot u \xi^{2}
$$

zu setzen, wo $C_{t}^{2}$ die binäre Form 2. Ordnung ist, deren Nullpunkte die Doppelelemente der Involution angeben, und $\xi$ den Scheitel des Strahlenbüschels vorstellt, welchem die Linienpaare derselben angehören.

Durch Einführung dieser Voraussetzungen in die Gleichung (4) erhält man jetzt:

$$
\left(C_{t}^{2}\right)^{2} \cdot u_{\xi}^{2} v_{\xi}^{2}\left[\left(\alpha_{t} a_{z} a_{y}\right)^{2}-c \cdot \alpha_{t} a_{x}^{2} \cdot \alpha_{t} a_{y}^{2}\right]=0 .
$$


Es bleibt also nur noch eine quadratische Gleichung für $t$, entsprechend dem Werthe 2 der Charakteristik $\lambda$. Diese Gleichung reducirt sich, sobald $c=1$ wird, ebenfalls auf $C_{t}^{2}=0$, da der Ausdruck in der Klammer, der von $\xi$ ganz unabhängig ist, alsdann die Form

$$
\left(a_{x} a_{y}\right)^{2}-a_{x}^{2} \cdot a_{y}^{2}
$$

annimmt, und daher für jede Doppellinie, aber nicht für jedes Linienpaar identisch verschwindet.

Das Kegelschnittsystem (2) hat also mit jeder Involution von Punktepaaren oder Strahlenpaaren nur deren Doppelelemente gemein.

Es ist ersichtlich, dass eine Theorie, welche den Begriff der eigentlichen Lösung des Charakteristikenproblems von der Beweglichkeit derselben abhängig macht, den Charakteristiken $\lambda$ und $\lambda^{\prime}$ eines vierfach ausgedehnten Systems die einfache Deutung, welche dieselben hier gefunden haben, wenigstens allgemein nicht geben kann, da die Involution von Strahlen- und Punktepaaren gerade solche Systeme von Kegelschnitten sind, für welche, - soferne man sie überhaupt zulassen will - der Chasles'sche Satz in der Auffassung jener Theorie keine allgemeine Geltung mehr hat.

Leipzig, im Juli 1885. 